ESAIM: PROCEEDINGS AND SURVEYS, March 2016, Vol. 53, p. 1-21

M. Campos Pinto and F. Charles, Editors

\title{
A NUMERICAL STUDY OF THE SOLUTION OF X-MODE EQUATIONS AROUND THE HYBRID RESONANCE*
}

\author{
CÉline Caldini-Queiros ${ }^{1}$, Bruno Després ${ }^{2}$, Lise-Marie Imbert-Gérard ${ }^{3}$ And \\ MARYNA KACHANOVSKA ${ }^{4}$
}

\begin{abstract}
The hybrid resonance is a physical phenomenon that appears for example in the heating of plasma, and as such is of scientific interest for the development of the ITER project. In this paper we focus on solutions of low regularity to Maxwell equations in magnetized plasmas. Our main purpose is three-fold. First, we aim at investigating the finite element approximation of the frequency-domain problem. Second, we would like to study the resonant solutions in the time domain, with the help of two different finite difference approximations. We finally compare the results with the ones obtained in the frequency domain, by numerical examination of the limiting absorption and limited amplitude principles.

Résumé. La résonnance hybride est un phénomène physique qui apparait par exemple lorsque l'on chauffe un plasma. Il est en particulier d'intérêt scientifique dans le cadre du développement du projet ITER. Dans ce papier, nous nous concentrons sur des solutions faiblement régulières des équations de Maxwell pour les plasmas magnétiques. Notre but est ici triple. Dans un premier temps, nous approchons numériquement la formulation fréquentielle à l'aide d'éléments finis. Dans un deuxième temps, nous étudions les solutions résonnantes dans le domaine temporel, à l'aide de deux méthodes distinctes de différences finies. Finalement, nous comparons numériquement les solutions fréquentielles avec le comportement en temps long des solutions temporelles, dans le cadre des principes d'amplitude et d'absorption limite.
\end{abstract}

\section{INTRODUCTION}

Modeling various phenomena in plasmas is of practical importance for developing new sources of energy based on plasma fusion, see the ITER project ${ }^{1}$. This article concentrates on studying a phenomenon of hybrid resonance [22, which is observed in experiments (see 66,7,11]) and is described mathematically as the nonregularity of the solutions of Maxwell equations in magnetized plasmas 10. Physically the hybrid resonance corresponds to situations where the electric field becomes infinite due to some resonance coupling between the

\footnotetext{
* The authors acknowledge the support of ANR under contract ANR-12-BS01-0006-01. Moreover, this work was carried out within the framework of the European Fusion Development Agreement and the French Research Federation for Fusion Studies. It is supported by the European Communities under the contract of Association between Euratom and CEA. The views and opinions expressed herein do not necessarily reflect those of the European Commission.

${ }^{1}$ Max Planck Institute für PlasmaPhysik, Garching bei München, Germany

2 Sorbonne Universités, UPMC Univ Paris 06, UMR 7598, Laboratoire Jacques-Louis Lions, F-75005, Paris, France

${ }^{3}$ Courant Institute of Mathematical Sciences, New York University

${ }^{4}$ POEMS, INRIA, ENSTA ParisTech, Palaiseau, France

$1_{\text {www.iter.org }}$
}

(C) EDP Sciences, SMAI 2016 
background magnetic field and the polarization of the wave. The energy deposit is resonant and may exceed by far the energy exchange which occurs in Landau damping, see 15. Contrary to the Landau damping, however, hybrid resonance appears in a simpler one-dimensional model coupling fluid equations with the non-electrostatic part of Maxwell equations. Since the mathematics of hybrid resonance is far to be understood in dimension greater or equal to two, we restrict this work to dimension one.

To illustrate the hybrid resonance, we consider in this work a time-dependent wave propagation problem leading to the cold plasma model for a single species (electrons) under a uniform in time background magnetic field $\mathbf{B}_{0}=\left(0,0, B_{0}\right)$. We restrict our attention to the poloidal plane, which is the plane perpendicular to the direction of the magnetic field. The model can be described by the time-dependent Maxwell system 22

$$
\begin{array}{r}
-\epsilon_{0} \partial_{t} E_{x}+\partial_{y} H_{z}=J_{x}, \\
-\epsilon_{0} \partial_{t} E_{y}-\partial_{x} H_{z}=J_{y}, \\
\mu_{0} \partial_{t} H_{z}+\partial_{x} E_{y}-\partial_{y} E_{x}=0,
\end{array}
$$

coupled through the linear electronic current $\mathbf{J}=e N_{e}(\mathbf{r}) \mathbf{u}$ with the equations

$$
\begin{aligned}
& m_{e} \partial_{t} u_{x}=\epsilon_{0} e E_{x}+e B_{0}(\mathbf{r}) u_{y}-m_{e} \nu u_{x} \\
& m_{e} \partial_{t} u_{y}=\epsilon_{0} e E_{y}-e B_{0}(\mathbf{r}) u_{x}-m_{e} \nu u_{y}
\end{aligned}
$$

where $\nu \geq 0$ is the friction between particles, $e<0$ is the charge of electrons, $m_{e}$ the electron mass and $N_{e}$ the electron density. In the cold plasma model, because of the frequencies considered, the electron density depends on space and is uniform in time. A non-zero value of $\nu$ constitutes a basis for the limiting absorption principle. The unknowns are the electron velocity $\mathbf{u}$ and the electromagnetic fields. The energy of this system in a domain $\Omega \in \mathbb{R}^{2}$ can be expressed as, see $[10$,

$$
\mathcal{E}(t)=\int_{\Omega}\left(\frac{\epsilon_{0}|\mathbf{E}(t, \mathbf{r})|^{2}}{2}+\frac{|\mathbf{B}(t, \mathbf{r})|^{2}}{2 \mu_{0}}+\frac{m_{e}|\mathbf{J}(t, \mathbf{r})|^{2}}{2|e| N_{e}(\mathbf{r})}\right) \mathrm{d} \mathbf{r}
$$

and $(1,2)$ yield

$$
\frac{d}{d t} \mathcal{E}=-\nu\left(\left\|u_{x}\right\|_{L^{2}(\Omega)}^{2}+\left\|u_{y}\right\|_{L^{2}(\Omega)}^{2}\right)+\text { boundary terms. }
$$

To illustrate unexpected difficulties that can occur in this system, consider the frequency domain counterpart of 1 2 2 , which corresponds to the cold plasma model. We introduce the plasma frequency $\omega_{p}(\mathbf{r})=\sqrt{\frac{|e|^{2} N_{e}(\mathbf{r})}{m \epsilon_{0}}}$, the cyclotron frequency $\omega_{c}(\mathbf{r})=\frac{e B_{0}(\mathbf{r})}{m_{e}}$ and perform the Fourier transform in time of 1 2) (with the convention $\left.\partial_{t} \rightarrow-i \omega\right)$, for a particular case $\nu=0$ :

$$
\operatorname{curl} \operatorname{curl} \hat{\mathbf{E}}-\frac{\omega^{2}}{c^{2}} \underline{\underline{\varepsilon_{\omega}^{0}}}(\mathbf{r}) \hat{\mathbf{E}}=0
$$

where the cold-plasma dielectric tensor [22, Chapter 1-2]

$$
\begin{array}{r}
\underline{\underline{\varepsilon_{\omega}^{0}}}(\mathbf{r})=\left(\begin{array}{cc}
\alpha_{\omega}(\mathbf{r}) & -i \delta_{\omega}(\mathbf{r}) \\
i \delta_{\omega}(\mathbf{r}) & \alpha_{\omega}(\mathbf{r})
\end{array}\right), \\
\alpha_{\omega}(\mathbf{r})=1-\frac{\omega_{p}^{2}(\mathbf{r})}{\omega^{2}-\omega_{c}^{2}(\mathbf{r})}, \delta_{\omega}(\mathbf{r})=\frac{\omega_{c}(\mathbf{r}) \omega_{p}^{2}(\mathbf{r})}{\omega\left(\omega^{2}-\omega_{c}^{2}(\mathbf{r})\right)} .
\end{array}
$$

The point $\mathbf{r}_{0}$ s.t. $\omega_{c}\left(\mathbf{r}_{0}\right)=\omega$, i.e. when $\underline{\underline{\varepsilon_{\omega}^{0}}}\left(\mathbf{r}_{0}\right)$ is singular, is called a cyclotron resonance (c.f. 22, Chapter 1-5]). In 9 it was demonstrated for a 1D-counterpart of the above system that such points behave like removable singularities, hence this case is not of interest for the present work. 
The hybrid resonance is precisely defined where $\omega_{p}^{2}(\mathbf{r})+\omega_{c}^{2}(\mathbf{r})=\omega^{2}$ and $\omega_{c}(\mathbf{r}) \omega_{p}(\mathbf{r}) \neq 0$, i.e. when the diagonal part of the tensor $\underline{\underline{\varepsilon_{\omega}^{0}}}(\mathbf{r})$ vanishes and the non-diagonal part remains non-zero. As shown in 9,10 , for a 1D-counterpart of (3), in this case the time-harmonic electric field component $\hat{E}_{x}$ is not necessarily square integrable (for a demonstration of this behaviour with the help of a simpler example, namely the Budden problem, see e.g. [10]). This apparent paradox is of course the source of important numerical difficulties which are the subject of the present study.

Before proceeding, let us define the geometrical setting of the problem. We consider the frequency domain problem (3) posed in a truncated infinite half-plane, $(-L, H) \times \mathbb{R}$, for some $L>0$ and $H>0$, with the following boundary conditions. At the left boundary of the domain, which represents the wall of the Tokamak, we impose a Robin boundary condition

$$
\operatorname{curl} \hat{\mathbf{E}}+i \lambda \hat{\mathbf{E}} \wedge \mathbf{n}=\hat{g}_{i n c} \equiv \operatorname{curl} \mathbf{E}_{i n c}+i \lambda \mathbf{E}_{i n c} \wedge \mathbf{n},
$$

where $\mathbf{E}_{i n c}=\exp (i \lambda x)\left(\begin{array}{l}E_{1} \\ E_{2}\end{array}\right)$ and $\lambda \geq 0$ is the frequency of the antenna. At the right boundary we impose $\operatorname{curl} \hat{\mathbf{E}}=0$.

In this work we focus on stratified medium case: we suppose that the electron density $N_{e}$ and the magnetic field $B_{0}$ are uniform in the second coordinate $y, N_{e}=N_{e}(x)$ and $B_{0}=B_{0}(x)$, see e.g. 10. We additionally assume that $N_{e}>0$ on $[-L, H]$. We choose this simplified case to focus on the fundamental mathematical difficulties of the hybrid resonance [9, 10].

Under the aforementioned assumptions on $N_{e}$ and $B_{0}$, the one-dimensional model can be derived from (1) by performing the Fourier transform in $y\left(\partial_{y} \rightarrow i \theta, \theta \in \mathbb{R}\right)$ :

$$
\left\{\begin{array}{l}
\epsilon_{0} \partial_{t} E_{x}-i \theta H_{z}=-e N_{e} u_{x} \\
\epsilon_{0} \partial_{t} E_{y}+\partial_{x} H_{z}=-e N_{e} u_{y} \\
\mu_{0} \partial_{t} H_{z}+\partial_{x} E_{y}-i \theta E_{x}=0 \\
m_{e} \partial_{t} u_{x}=e E_{x}+e u_{y} B_{0}-\nu m_{e} u_{x} \\
m_{e} \partial_{t} u_{y}=e E_{y}-e u_{x} B_{0}-\nu m_{e} u_{y}
\end{array}\right.
$$

posed in the one-dimensional domain $(-L, H)$, with the corresponding boundary conditions:

$$
\begin{array}{r}
\left.\left(\partial_{x} E_{y}-i \theta E_{x}+i \lambda E_{y}\right)\right|_{x=-L}=g_{i n c}(t), \\
\left.\partial_{x} E_{y}\right|_{x=H}=0,
\end{array}
$$

and the initial conditions for values $\left.E_{x}\right|_{t=0},\left.u_{x}\right|_{t=0},\left.E_{y}\right|_{t=0},\left.u_{y}\right|_{t=0},\left.H_{z}\right|_{t=0}$. This is the time-domain model that will be considered in the rest of the article, unless stated otherwise.

The frequency domain formulation of (6) for $\nu=0$ is

$$
\left(\begin{array}{l}
i \theta \partial_{x} \hat{E}_{y}+\theta^{2} \hat{E}_{x} \\
i \theta \partial_{x} \hat{E}_{x}-\partial_{x}^{2} \hat{E}_{y}
\end{array}\right)-\frac{\omega^{2}}{c^{2}} \underline{\underline{\varepsilon_{\omega}^{0}}}(x)\left(\begin{array}{c}
\hat{E}_{x} \\
\hat{E}_{y}
\end{array}\right)=0
$$

see (4). Indeed, in a stratified medium, the coefficients $\alpha_{\omega}, \delta_{\omega}$ depend solely on $x$ and $\omega$. We concentrate on a physical situation of the hybrid resonance, which we define as in [9].

Definition 1.1 (of a hybrid resonance). Let $\omega \in \mathbb{R}$ be fixed. We will call a point $x_{h} \in \mathbb{R}$ a hybrid resonance if $\alpha_{\omega}$ has a simple root in $x_{h}$ and has no other roots in an $\epsilon$-vicinity of this point $B_{\epsilon}\left(x_{h}\right)$, for some $\epsilon>0$, and $\delta_{\omega}\left(x_{h}\right) \neq 0$. The parameter $\nu$ is at the same the physical friction and a regularization parameter. The hybrid resonance singular solution shows up at $x_{h}$ at the limit $\nu \rightarrow 0^{+}$. 


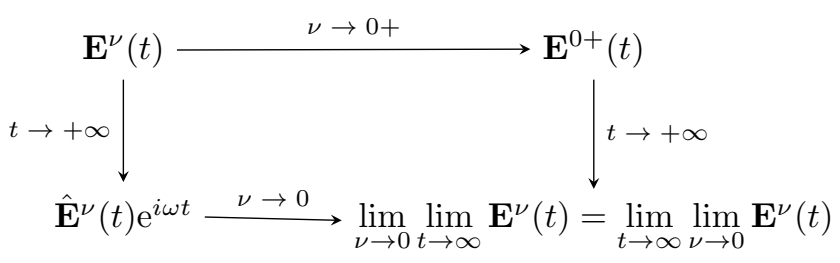

FIGURE 1. Schematic representation of the equivalence of the limited absorption and limiting amplitude principles.

To investigate the behaviour of the solutions at the hybrid resonance, we will study a sequence of the regularized problems, for $\nu \rightarrow 0$. These problems are not the frequency domain expression of (6), but a simple regularization of (8). This choice will be justified in further sections. The equations read:

$$
\left(\begin{array}{l}
i \theta \partial_{x} \hat{E}_{y}+\theta^{2} \hat{E}_{x} \\
i \theta \partial_{x} \hat{E}_{x}-\partial_{x}^{2} \hat{E}_{y}
\end{array}\right)-\frac{\omega^{2}}{c^{2}}\left(\underline{\underline{\varepsilon_{\omega}^{0}}}(x)+i \nu \mathrm{Id}\right)\left(\begin{array}{l}
\hat{E}_{x} \\
\hat{E}_{y}
\end{array}\right)=0
$$

where Id is the identity matrix.

The goal of this article is three-fold. First, we investigate the finite element approximation of the 1D frequency domain problem (9), with a regularized dielectric tensor for a small parameter $\nu$. We prove the well-posedness of this problem for $\nu \neq 0$ in Section 2.1 and demonstrate that the use of $P_{1}$ finite elements allows to approximate the singularity of the solution efficiently (Section 4.1). Second, we develop an original scheme based on widely appreciated semi-lagrangian schemes for the discretization of time domain Maxwell's equations with a linear current. Third, we consider the case $\nu \rightarrow 0$, and study the limiting amplitude solution $\lim _{t \rightarrow+\infty} \lim _{\nu \rightarrow 0} \mathbf{E}(t)$ obtained with the help of the FDTD discretization of (6), suggested in [5]. We compare this result with $\hat{\mathbf{E}} \mathrm{e}^{-i \omega t}$, computed in the frequency domain, for $\nu \rightarrow 0$, which corresponds to $\lim _{\nu \rightarrow 0} \lim _{t \rightarrow+\infty} \mathbf{E}(t)$. Such a comparison is a way to study the formal commutation relation between the limited absorption and the limiting amplitude principles. In application to a non-resonant case of (8), equipped with boundary conditions, the limited absorption principle states that the solution of $(9)$ would approach the solution of $(8)$ as $\nu \rightarrow 0$. Similarly, the limiting amplitude principle applied to the problem (6) states that if the boundary conditions are chosen harmonic in time, e.g. $\left.\partial_{x} E_{y}\right|_{x=-L}=\mathrm{e}^{-i \omega_{*} t}$, then the solution of this problem $(\mathbf{E}(t), H(t))$ asymptotically $(t \rightarrow+\infty)$ tends to a steady state of the form $\left(\hat{\mathbf{E}}\left(\omega_{*}\right), \hat{H}\left(\omega_{*}\right)\right) \mathrm{e}^{-i \omega_{*} t}$, where $\left(\hat{\mathbf{E}}\left(\omega_{*}\right), \hat{H}\left(\omega_{*}\right)\right)$ is the solution of 60 in the frequency domain, with $\omega=\omega_{*}$ and the boundary condition $\left.\partial_{x} \hat{E}_{y}\right|_{x=-L}=1$. For more details see e.g. 12,20. The commutation between the two principles then writes as $\lim _{\nu \rightarrow 0} \lim _{t \rightarrow+\infty}=\lim _{t \rightarrow+\infty} \lim _{\nu \rightarrow 0}$, see Figure 1. Even though it is true for standard linear wave problems, it is not clear if it still holds in the singular case of the hybrid resonance.

To our knowledge, such numerical studies have not been performed in the existing literature.

\section{FREQUENCY DOMAin STUDY}

This section reviews the connection between the frequency domain counterpart of the problem (6) and the regularized problem (9). Model (6) can be rewritten in the frequency domain as

$$
\begin{array}{r}
\left(\begin{array}{l}
i \theta \partial_{x} \widehat{E}_{y}+\theta^{2} \widehat{E}_{x} \\
i \theta \partial_{x} \widehat{E}_{x}-\partial_{x}^{2} \widehat{E}_{y}
\end{array}\right)-\frac{\omega^{2}}{c^{2}} \underline{\underline{\varepsilon_{\omega}^{\nu}}}(x)\left(\begin{array}{c}
\widehat{E}_{x} \\
\widehat{E}_{y}
\end{array}\right)=0, \\
\underline{\underline{\varepsilon_{\omega}^{\nu}}}(x)=\left(\begin{array}{cc}
\alpha_{\omega}^{\nu}(x) & -i \delta_{\omega}^{\nu}(x) \\
i \delta_{\omega}^{\nu}(x) & \alpha_{\omega}^{\nu}(x)
\end{array}\right), \quad \alpha_{\omega}^{\nu}(x)=1-\frac{\omega_{\nu} \omega_{p}^{2}(x)}{\omega\left(\omega_{\nu}^{2}-\omega_{c}^{2}(x)\right)}, \quad \delta_{\omega}^{\nu}(x)=\frac{\omega_{c}(\mathbf{r}) \omega_{p}^{2}(x)}{\omega\left(\omega_{\nu}^{2}-\omega_{c}^{2}(x)\right)},
\end{array}
$$


where the frequency $\omega_{\nu}=\omega+i \nu$ is shifted in the complex plane. The choice of the non-zero plasma friction $\nu>0$ is a regularization of the problem. A series expansion as $\nu \rightarrow 0$ gives at leading orders

$$
\begin{gathered}
\alpha_{\omega}^{\nu}=\alpha_{\omega}+i \nu \frac{\omega_{p}^{2}\left(\omega^{2}+\omega_{c}^{2}\right)}{\omega\left(\omega^{2}-\omega_{c}^{2}\right)^{2}}=\alpha_{\omega}+i \nu D_{\alpha}, \\
\delta_{\omega}^{\nu}=\delta_{\omega}-i \nu \frac{2 \omega_{c} \omega_{p}^{2}}{\left(\omega^{2}-\omega_{c}^{2}\right)^{2}}=\delta_{\omega}+i \nu D_{\delta}
\end{gathered}
$$

where $\alpha_{\omega}, \delta_{\omega}$ are defined by (4). One thus can consider the following regularization of the cold plasma dielectric tensor of the above problem, valid as $\nu \rightarrow 0$ :

$$
\underline{\underline{\varepsilon_{\omega}^{\nu}}} \approx \underline{\underline{\varepsilon_{\omega}^{0}}}+i \nu\left(\begin{array}{cc}
D_{\alpha} & -D_{\delta} \\
D_{\delta} & D_{\alpha}
\end{array}\right)
$$

However, as demonstrated in [9] for the 1D setting, a significantly simpler regularization (considered in [10])

$$
\underline{\underline{\varepsilon_{\omega}^{\nu}}}(x) \approx \underline{\underline{\varepsilon_{\omega}^{0}}}(x)+i \nu \mathrm{Id}
$$

yields the same limit solution as $\nu \rightarrow 0$. As a consequence we will consider in this work the simpler regularized system

$$
\begin{gathered}
\left(\begin{array}{c}
i \theta \partial_{x} \widehat{E}_{y}+\theta^{2} \widehat{E}_{x} \\
i \theta \partial_{x} \widehat{E}_{x}-\partial_{x}^{2} \widehat{E}_{y}
\end{array}\right)-\frac{\omega^{2}}{c^{2}}\left(\underline{\underline{\varepsilon_{\omega}^{0}}}(x)+i \nu \mathrm{Id}\right)\left(\begin{array}{l}
\widehat{E}_{x} \\
\widehat{E}_{y}
\end{array}\right)=0 \\
\underline{\underline{\varepsilon_{\omega}^{0}}}(x)=\left(\begin{array}{cc}
\alpha_{\omega}(x) & -i \delta_{\omega}(x) \\
i \delta_{\omega}(x) & \alpha_{\omega}(x)
\end{array}\right) .
\end{gathered}
$$

For brevity, from now on we will denote $\frac{\omega^{2}}{c^{2}} \alpha_{\omega}, \frac{\omega^{2}}{c^{2}} \delta_{\omega}$ by $\alpha, \delta$ and consider a sequence of regularized problems

$$
\begin{aligned}
& \left(\begin{array}{l}
i \theta \partial_{x} \widehat{E}_{y}+\theta^{2} \widehat{E}_{x} \\
i \theta \partial_{x} \widehat{E}_{x}-\partial_{x}^{2} \widehat{E}_{y}
\end{array}\right)-\left(\underline{\underline{\varepsilon}}^{0}(x)+i \nu \mathrm{Id}\right)\left(\begin{array}{c}
\widehat{E}_{x} \\
\widehat{E}_{y}
\end{array}\right)=0, \\
& \underline{\underline{\varepsilon}}^{0}(x)=\left(\begin{array}{cc}
\alpha(x) & -i \delta(x) \\
i \delta(x) & \alpha(x)
\end{array}\right),
\end{aligned}
$$

where $\alpha, \delta$ satisfy the following assumption.

Assumption 2.1. The coefficients $\alpha(x)$ and $\delta(x)$ are assumed to be sufficiently smooth, i.e. continuous in $[-L, H]$.

This assumption can be ensured, for example, by requiring the continuity of $N_{e}(x), B_{0}(x)$ in $[-L, H]$ and the absence of the cyclotron resonance, i.e. for all $x\left|\omega_{c}^{2}(x)-\omega^{2}(x)\right|>c>0$ on this interval.

Remark 2.2. For the time domain problem (6) the choice of the sign of $\nu>0$ is of crucial importance for the stability of the problem. In the frequency domain, as we can see from the above and from [10, 18], the sign of the parameter $\nu$ can be chosen arbitrarily to regularize the problem. However, as demonstrated in the aforementioned works, as $\nu \rightarrow 0 \pm$, the corresponding limiting solutions differ, though both exhibit the singularity in the point of the hybrid resonance. 


\subsection{Well-Posedness of the Frequency Domain Problem}

In this section we study the well-posedness of Problem (10) with the boundary conditions (5), thanks to the Lax-Milgram lemma. After introducing $\Omega=(-L, H) \subset \mathbb{R}$ and defining the function space $\mathbf{V}=L^{2}(\Omega) \times H^{1}(\Omega)$, equipped with the norm

$$
\|\mathbf{v}\|_{\mathbf{V}}^{2}=\left\|v_{1}\right\|_{L^{2}(\Omega)}^{2}+\left\|v_{2}\right\|_{H^{1}(\Omega)}^{2} \quad \text { for } \mathbf{v}=\left(v_{1}, v_{2}\right),
$$

the resulting one-dimensional system reads in the following variational form:

$$
\begin{array}{r}
\int_{-L}^{H}\left(E_{y}^{\prime}-i \theta E_{x}\right) \overline{\left(\widetilde{E}_{y}^{\prime}-i \theta \widetilde{E}_{x}\right)}-\int_{-L}^{H}\left(\underline{\underline{\varepsilon}}^{0}+i \nu \mathrm{Id}\right) \mathbf{E} \cdot \overline{\widetilde{\mathbf{E}}}-i \lambda E_{y}(-L) \overline{\widetilde{E}_{y}(-L)}=-g_{i n c}(-L) \overline{\left(\widetilde{E}_{y}(-L)\right)}, \\
\text { for all } \widetilde{\mathbf{E}}=\left(\widetilde{E}_{x}, \widetilde{E}_{y}\right) \in \mathbf{V} .
\end{array}
$$

Recall that here $\lambda \geq 0$.

Let us remark that in this and further sections, where it is not ambiguous, we will use the notation $E_{x, y}$ instead of $\widehat{E}_{x, y}$ for convenience. Also, by $\|u\|_{L^{2}}$ we will denote $\|u\|_{L^{2}(\Omega)}$, for $u \in L^{2}(\Omega)$, and $\|\mathbf{u}\|_{L^{2}(\Omega)}=$ $\left(\left\|u_{1}\right\|_{L^{2}}^{2}+\left\|u_{2}\right\|_{L^{2}}^{2}\right)^{\frac{1}{2}}$, for $\mathbf{u}=\left(u_{1}, u_{2}\right) \in\left(L^{2}(\Omega)\right)^{2}$.

For the sake of conciseness, we introduce the following notation. Let $a$ and $l$ be the bilinear and linear forms, defined for $\mathbf{u}, \mathbf{v} \in \mathbf{V}$ :

$$
a(\mathbf{u}, \mathbf{v})=a_{1}(\mathbf{u}, \mathbf{v})+i a_{2}(\mathbf{u}, \mathbf{v}) \text { and } l(\mathbf{v})=-g_{i n c}(-L) \overline{\left(v_{2}(-L)\right)}
$$

where $a_{1}=a_{1}^{*}$ and $a_{2}=a_{2}^{*}$ are hermitian

$$
\left\{\begin{array}{l}
a_{1}(\mathbf{u}, \mathbf{v})=\int_{-L}^{H}\left(u_{2}^{\prime}-i \theta u_{1}\right) \overline{\left(v_{2}^{\prime}-i \theta v_{1}\right)}-\int_{-L}^{H} \underline{\underline{\varepsilon}}^{0} \mathbf{u} \cdot \overline{\mathbf{v}} \\
a_{2}(\mathbf{u}, \mathbf{v})=-\nu \int_{-L}^{H} \mathbf{u} \cdot \overline{\mathbf{v}}-\lambda u_{2}(-L) \overline{v_{2}(-L)}
\end{array}\right.
$$

Then 12 reads: find $\mathbf{E}$ such that

$$
a(\mathbf{E}, \tilde{\mathbf{E}})=l(\tilde{\mathbf{E}}), \text { for all } \tilde{\mathbf{E}} \in \mathbf{V} .
$$

Let us denote the spectral radius of $\underline{\underline{\varepsilon}}^{0}$ by $\hat{\varepsilon}=\left\|\rho\left(\underline{\underline{\varepsilon}}^{0}\right)\right\|_{L^{\infty}(\Omega)}$. By Assumption 2.1, the spectral radius $\hat{\varepsilon}=\max \left(\|\alpha+\delta\|_{L^{\infty}(\Omega)},\|\alpha-\delta\|_{L^{\infty}(\Omega)}\right)$ is bounded.

We now prove the following result.

Lemma 2.3. Let Assumption 2.1 hold. Then the bilinear form

$$
a(\mathbf{u}, \mathbf{v}): \mathbf{V} \times \mathbf{V} \rightarrow \mathbb{C}
$$

is continuous and coercive for all $\lambda \geq 0$ and $\nu>0$ : for all $\mathbf{u}, \mathbf{v} \in \mathbf{V}$ it holds that

$$
\left\{\begin{array}{l}
\Re\left(\mathrm{e}^{i \beta_{\nu}} a(\mathbf{u}, \mathbf{u})\right) \geq \frac{|\nu|}{\sqrt{\left(\hat{\varepsilon}+\theta^{2}+1\right)^{2}+\nu^{2}}}\left(\frac{1}{2}\left\|u_{2}^{\prime}\right\|_{L^{2}}^{2}+\|\mathbf{u}\|_{L^{2}}^{2}\right), \text { for some } \beta_{\nu} \in\left(0, \frac{\pi}{2}\right), \\
|a(\mathbf{u}, \mathbf{v})| \leq C\|\mathbf{u}\|_{\mathbf{v}}\|\mathbf{v}\|_{\mathbf{v}}
\end{array}\right.
$$

where $C>0$ does not depend on $\nu$. Problem (12) is well-posed in $\mathbf{V}$.

Proof. The boundedness of $a$ is obvious using the continuous embedding in dimension one $H^{1}(\Omega) \subset C^{0}(\bar{\Omega})$. Let us focus on the proof of coercivity. Given $\hat{\varepsilon}$ the spectral radius of $\underline{\underline{\varepsilon}}^{0}, \hat{\varepsilon} \mathrm{Id}-\underline{\underline{\varepsilon}}^{0}$ is a non-negative hermitian matrix. Therefore

$$
a_{1}(\mathbf{u}, \mathbf{u})+\hat{\varepsilon}\|\mathbf{u}\|_{L^{2}}^{2}=\left\|u_{2}^{\prime}-i \theta u_{1}\right\|_{L^{2}}^{2}+\left(\left(\hat{\varepsilon} \mathrm{Id}-\underline{\underline{\varepsilon}}^{0}\right) \mathbf{u}, \overline{\mathbf{u}}\right) \geq\left\|u_{2}^{\prime}-i \theta u_{1}\right\|_{L^{2}}^{2} \geq \frac{1}{2}\left\|u_{2}^{\prime}\right\|_{L^{2}}^{2}-\theta^{2}\left\|u_{1}\right\|_{L^{2}}^{2} .
$$


Thus $a_{1}(\mathbf{u}, \mathbf{u}) \geq \frac{1}{2}\left\|u_{2}^{\prime}\right\|_{L^{2}}^{2}-\left(\hat{\varepsilon}+\theta^{2}\right)\|\mathbf{u}\|_{L^{2}}^{2}$. On the other hand, for $\lambda \geq 0, a_{2}(\mathbf{u}, \mathbf{u}) \leq-\nu\|\mathbf{u}\|_{L^{2}}^{2}$. Therefore

$$
a_{1}(\mathbf{u}, \mathbf{u})-\frac{\hat{\varepsilon}+\theta^{2}+1}{\nu} a_{2}(\mathbf{u}, \mathbf{u}) \geq \frac{1}{2}\left\|u_{2}^{\prime}\right\|_{L^{2}}^{2}+\|\mathbf{u}\|_{L^{2}}^{2},
$$

or, introducing $\beta_{\nu}=\arccos \frac{1}{\sqrt{1+\left(\hat{\varepsilon}+\theta^{2}+1\right)^{2} \nu^{-2}}}$, this is equivalent to

$$
\Re\left(\mathrm{e}^{i \beta_{\nu}} a(\mathbf{u}, \mathbf{u})\right) \geq \frac{1}{\sqrt{1+\left(\hat{\varepsilon}+\theta^{2}+1\right)^{2} \nu^{-2}}}\left(\frac{1}{2}\left\|u_{2}^{\prime}\right\|_{L^{2}}^{2}+\|\mathbf{u}\|_{L^{2}}^{2}\right) \geq \frac{\nu}{\sqrt{\left(\hat{\varepsilon}+\theta^{2}+1\right)^{2}+\nu^{2}}}\left(\frac{1}{2}\left\|u_{2}^{\prime}\right\|_{L^{2}}^{2}+\|\mathbf{u}\|_{L^{2}}^{2}\right) .
$$

Problem 12 is then uniquely solvable thanks to the Lax-Milgram theorem.

Remark 2.4. The above proof is not optimal when the diagonal coefficient is uniformly positive, namely $\alpha(x)>$ $c$ for some $c>0$ on the whole interval $[-L, H]$, at least for the case $\theta=0$. In this particular case Problem (12) is well-posed for $\nu=0$. More precisely, it can be written as a system of two equations, the first equation (for $E_{y}$ ) being the variational formulation for the Helmholtz equation with the variable coefficient $k^{2}=\left(\alpha-\frac{\delta^{2}}{\alpha}\right)$ coupled to boundary conditions (7), and the second equation being $\left(E_{x}, u\right)=\left(\frac{i \delta}{\alpha} E_{y}, u\right)$ for all $u \in L^{2}(\Omega)$. The demonstration of the well-posedness of the former problem, with additional assumptions on boundary conditions and smoothness of the coefficients, can be found in [18. Theorem 5.2.3]. Provided the solution $E_{y} \in H^{1}(\Omega)$, $E_{x} \in L^{2}(\Omega)$ is uniquely defined from the latter equation.

\subsection{Discretization of the Frequency Domain Problem}

Testing the variational formulation $\sqrt{12}$ with $\left(\tilde{E}_{x}, 0\right)$ and $\left(0, \tilde{E}_{y}\right) \in \mathbf{V}$ and using an explicit expression for the tensor of the dielectric permittivity (11), we get the following system of two equations

$$
\begin{gathered}
i \theta \int_{-L}^{H}\left(E_{y}^{\prime}-i \theta E_{x}\right) \overline{\tilde{E}_{x}}-\int_{-L}^{H}\left((\alpha+i \nu) E_{x}-i \delta E_{y}\right) \overline{\tilde{E}_{x}}=0 \\
\int_{-L}^{H}\left(E_{y}^{\prime}-i \theta E_{x}\right) \tilde{E}_{y}^{\prime}-\int_{-L}^{H}\left(i \delta E_{x}+(\alpha+i \nu) E_{y}\right) \overline{\tilde{E}}_{y}-i \lambda E_{y}(-L) \overline{\tilde{E}_{y}(-L)}=-g_{i n c}(-L) \overline{\left(\tilde{E}_{y}(-L)\right)}, \\
\text { for all } \tilde{E}_{x} \in L^{2}(\Omega), \tilde{E}_{y} \in H^{1}(\Omega) .
\end{gathered}
$$

Let us introduce two function spaces $V_{E_{x}} \subset L^{2}(\Omega)$ spanned by a set of basis functions $\left\{\psi_{j}\right\}_{j=1}^{N_{1}}$ and $V_{E_{y}} \subset H^{1}(\Omega)$ spanned by $\left\{\phi_{i}\right\}_{i=1}^{N_{2}}$. We discretize problem 13 as:

$$
E_{x} \approx \sum_{k=1}^{N_{1}} e_{x k} \psi_{k}, \quad E_{y} \approx \sum_{k=1}^{N_{2}} e_{y k} \phi_{k}
$$


Introducing

$$
\begin{array}{r}
\left(K^{\psi, \phi^{\prime}}\right)_{m k}=\int_{-L}^{H} \bar{\psi}_{m} \phi_{k}^{\prime} d x, \quad\left(M^{\psi}\right)_{m k}=\int_{-L}^{H} \psi_{k} \bar{\psi}_{m} d x, \quad\left(M^{\alpha, \psi}\right)_{m k}=\int_{-L}^{H}(\alpha(x)+i \nu) \psi_{m} \bar{\psi}_{k} d x, \\
\left(M^{\delta, \psi, \phi}\right)_{m k}=\int_{-L}^{H} \delta(x) \bar{\psi}_{m} \phi_{k} d x, \quad K_{\ell k}=\int_{-L}^{H} \phi_{k}^{\prime}(x) \bar{\phi}_{\ell}^{\prime}(x) d x, \quad\left(M^{\alpha, \phi}\right)_{\ell k}=\int_{-L}^{H}(\alpha(x)+i \nu) \bar{\phi}_{\ell} \phi_{k} d x, \\
I_{k m}^{\Gamma}=\bar{\phi}_{m}(-L) \phi_{k}(-L), \\
\boldsymbol{e}_{x}=\left(e_{x 1}, \ldots, e_{x N_{1}}\right)^{T}, \boldsymbol{e}_{y}=\left(e_{y 1}, \ldots, e_{y N_{2}}\right)^{T}, \\
\mathbf{0}_{n} \text { is an } n \text {-dimensional zero column vector, }
\end{array}
$$

we rewrite System $(13)$ in an antisymmetric block form:

$$
\left(\begin{array}{cc}
\theta^{2} M^{\psi}-M^{\alpha, \psi} & i \theta K^{\psi, \phi^{\prime}}+i M^{\delta, \psi, \phi} \\
-i \theta\left(K^{\psi, \phi^{\prime}}\right)^{*}-i\left(M^{\delta, \psi, \phi}\right)^{*} & K-M^{\alpha, \phi}-i \lambda I^{\Gamma}
\end{array}\right)\left(\begin{array}{c}
\boldsymbol{e}_{x} \\
\boldsymbol{e}_{y}
\end{array}\right)=-g_{i n c}(-L)\left(\begin{array}{c}
\mathbf{0}_{N_{1}} \\
\bar{\phi}_{1}(-L) \\
\bar{\phi}_{2}(-L) \\
\vdots \\
\bar{\phi}_{N_{2}}(-L)
\end{array}\right)
$$

This expression greatly simplifies when choosing $V_{E_{x}}=V_{E_{y}}$, spanned by a unique set of basis functions $\left(\phi_{m}\right)_{m=1}^{N_{2}}$, and in the case $\theta=0$. One obtains the matrix structure

$$
\left(\begin{array}{cc}
M^{\alpha, \phi} & -i M^{\delta, \phi, \phi} \\
-i\left(M^{\delta, \phi, \phi}\right)^{*} & K-M^{\alpha, \phi}-i \lambda I^{\Gamma}
\end{array}\right)\left(\begin{array}{c}
\boldsymbol{e}_{x} \\
\boldsymbol{e}_{y}
\end{array}\right)=-g_{i n c}(-L)\left(\begin{array}{c}
\mathbf{0}_{N_{1}} \\
\bar{\phi}_{1}(-L) \\
\bar{\phi}_{2}(-L) \\
\vdots \\
\bar{\phi}_{N_{2}}(-L)
\end{array}\right) .
$$

In this work we use the above formulation with the Lagrange $P_{1}$ basis elements.

\section{Time-Dependent Problem Discretization}

We develop hereafter the discretization of the time-dependent one-dimensional model (6), for the particular case $\theta=0$. We first show how this system can be discretized thanks to a Yee scheme suggested in [5], then a scheme based on semi-lagrangian discretization is employed.

\subsection{A staggered scheme}

We compute a numerical approximation of (6) with $\theta=0$ on a cartesian grid using the Yee scheme, as suggested in 5. Let $n$ and $\Delta t$ denote the time step index and the time step, as usual the discrete time is $t_{n}=n \Delta t$. It is standard (see [5, 24]) to discretize the electrical field on the integer time steps, whereas the field 
$H_{z}$ on half time steps, $t_{n+1 / 2}$. The time discretization of the system reads:

$$
\left\{\begin{array}{l}
\epsilon_{0} \frac{E_{x}^{n+1}-E_{x}^{n}}{\Delta t}=-e N_{e} \frac{u_{x}^{n+1}+u_{x}^{n}}{2} \\
\epsilon_{0} \frac{E_{y}^{n+1}-E_{y}^{n}}{\Delta t}+\partial_{x} H_{z}^{n+1 / 2}=-e N_{e} \frac{u_{y}^{n+1}+u_{y}^{n}}{2} \\
\mu_{0} \frac{H_{z}^{n+1 / 2}-H_{z}^{n-1 / 2}}{\Delta t}+\partial_{x} E_{y}^{n}=0 \\
m_{e} \frac{u_{x}^{n+1}-u_{x}^{n}}{\Delta t}=e \frac{E_{x}^{n+1}+E_{x}^{n}}{2}+e \frac{u_{y}^{n+1}+u_{y}^{n}}{2} B_{0}-\nu m_{e} \frac{u_{x}^{n+1}+u_{x}^{n}}{2} \\
m_{e} \frac{u_{y}^{n+1}-u_{y}^{n}}{\Delta t}=e \frac{E_{y}^{n+1}+E_{y}^{n}}{2}-e \frac{u_{x}^{n+1}+u_{x}^{n}}{2} B_{0}-\nu m_{e} \frac{u_{y}^{n+1}+u_{y}^{n}}{2}
\end{array}\right.
$$

In the Yee scheme, the different components of the vector fields are defined on staggered points. We summarize this discretization on Fig. 2 where the positions of $E_{x}, u_{x}$ (black dots), $E_{y}, u_{y}$ (blue dots) and $H_{z}$ (red dots) on the discretized grid in space and time are displayed. The fully discretized system then reads

$$
\begin{gathered}
\varepsilon_{0} \frac{\left.E_{x}\right|_{i} ^{n+1}-\left.E_{x}\right|_{i} ^{n}}{\Delta t}=-e N_{e} \frac{\left.u_{x}\right|_{i} ^{n+1}+\left.u_{x}\right|_{i} ^{n}}{2}, \\
\mu_{0} \frac{\left.E_{y}\right|_{i+1 / 2} ^{n+1}-\left.E_{y}\right|_{i+1 / 2} ^{n}+\frac{\left.H_{z}\right|_{i+1} ^{n+1 / 2}-\left.H_{z}\right|_{i} ^{n+1 / 2}}{\Delta t}=-e N_{e} \frac{\left.u_{y}\right|_{i+1 / 2} ^{n+1}+\left.u_{y}\right|_{i+1 / 2} ^{n}}{2}-\left.H_{z}\right|_{i} ^{n-1 / 2}}{\Delta t}+\frac{\left.E_{y}\right|_{i+1 / 2} ^{n}-\left.E_{y}\right|_{i-1 / 2} ^{n}}{\Delta x}=0 \\
m_{e} \frac{\left.u_{x}\right|_{i} ^{n+1}-\left.u_{x}\right|_{i} ^{n}}{\Delta t}=e \frac{\left.E_{x}\right|_{i} ^{n+1}+\left.E_{x}\right|_{i} ^{n}}{2}-\nu m_{e} \frac{\left.u_{x}\right|_{i} ^{n+1}+\left.u_{x}\right|_{i} ^{n}}{2}+e B_{0} \frac{\left.u_{y}\right|_{i+1 / 2} ^{n}+\left.u_{y}\right|_{i+1 / 2} ^{n+1}}{2} \\
m_{y} \frac{u_{i+1 / 2}^{n+1}-\left.u_{y}\right|_{i+1 / 2} ^{n}}{\Delta t}=e \frac{\left.E_{y}\right|_{i+1 / 2} ^{n+1}+\left.E_{y}\right|_{i+1 / 2} ^{n}}{2}-\nu m_{e} \frac{\left.u_{y}\right|_{i+1 / 2} ^{n+1}+\left.u_{y}\right|_{i+1 / 2} ^{n}}{2}-e B_{0} \frac{\left.u_{x}\right|_{i} ^{n}+\left.u_{x}\right|_{i} ^{n+1}}{2}
\end{gathered}
$$

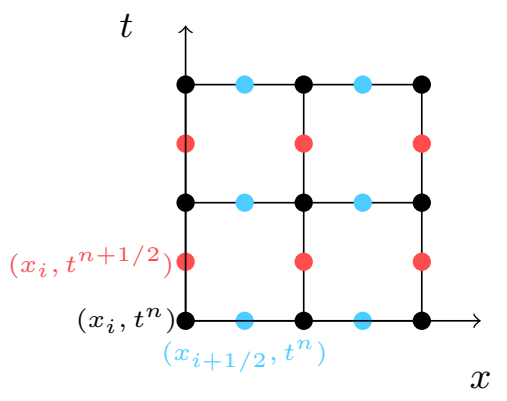

Figure 2. Positions of $E_{x}, u_{x}$ (black dots), $E_{y}, u_{y}$ (blue dots) and $H_{z}$ (red dots) on the discretized grid in space and time

It is straightforward to rewrite this system in an explicit form (we omit here these simple but slightly tedious computations).

\subsection{A co-localized scheme based on semi-lagrangian discretisation}

The scheme described hereafter is mentioned since it is based on some well-known numerical methods in plasma physics, the so-called semi-lagrangian schemes widely used for the numerical approximation of transport equations. It seems to be an original use of semi-lagrangian schemes and is mentioned as possible other option. We restrict the presentation to the minimum and leave the details of the construction to the alert reader. Concerning the vast literature on the use of semi-lagrangian schemes in numerical plasma physics, we refer the 
reader to $13,14,16,21,23$ and references therein. The structure of the following algorithm is very similar to the one developed in $[8]$ and can be adapted and studied numerically in any dimension on a Cartesian grid and for more complex right-hand sides. So mathematical properties of the first step of the following semi-lagrangian scheme have been described in 3 .

The idea is to split System (6) into a series of transport equations (here just 2) and a local in-the-cell system.

- At time step $t_{n}=n \Delta t$, all quantities are given $\left(E_{x}, E_{y}, H_{z}, u_{x}, u_{y}\right)_{i}^{n}$ in the cells, that is for all $i$ in the computational domain.

- The first step is to solve

$$
\left\{\begin{array}{l}
\epsilon_{0} \partial_{t} E_{x}=0 \\
\epsilon_{0} \partial_{t} E_{y}+\partial_{x} H_{z}=0 \\
\mu_{0} \partial_{t} H_{z}+\partial_{x} E_{y}=0 \\
m_{e} \partial_{t} u_{x}=0 \\
m_{e} \partial_{t} u_{y}=0
\end{array}\right.
$$

Since $\epsilon_{0} \mu_{0} c^{2}=1$, this can be performed solving two transport equations

$$
\begin{cases}\partial_{t} w+c \partial_{x} w=0, & w=E_{y}+c H_{z} \\ \partial_{t} z-c \partial_{x} z=0 & z=E_{y}-c H_{z}\end{cases}
$$

In our case we used a semi-lagrangian scheme for a time step $\Delta t$. In the tests, we used Strang's stencils described in [3] with CFL $\nu=0.5$, at order 7 . It yields the chain $\left(E_{y}, H_{z}\right)_{i}^{n} \mapsto(w, z)_{i}^{n} \mapsto(w, z)_{i}^{n *} \mapsto\left(E_{y}, H_{z}\right)_{i}^{n *}$. Here $n *$ denotes the intermediate time step of the algorithm.

- The second (and final) step is to solve

$$
\left\{\begin{array}{l}
\epsilon_{0} \partial_{t} E_{x}=-e N_{e} u_{x} \\
\epsilon_{0} \partial_{t} E_{y}=-e N_{e} u_{y} \\
\mu_{0} \partial_{t} H_{z}=0 \\
m_{e} \partial_{t} u_{x}=e E_{x}+e u_{y} B_{0}-\nu m_{e} u_{x} \\
m_{e} \partial_{t} u_{y}=e E_{y}-e u_{x} B_{0}-\nu m_{e} u_{y}
\end{array}\right.
$$

This is performed locally in every cell starting from the data at the preceding step. In our case we just adapt the algorithm developed for the staggered scheme. We make sure that the method is conservative in total energy if $\nu=0$, using a half-step discretization of the right-hand sides.

- Therefore all quantities are now given $\left(E_{x}, E_{y}, H_{z}, u_{x}, u_{y}\right)_{i}^{n+1}$ in the cells at time step $t_{n+1}=(n+1) \Delta t$, and that defines totally a time step of the algorithm.

\section{NumericAl EXPERIMENTS}

This section is organized as follows. The first part is dedicated to the numerical implementation and simulation of the frequency domain formulation (12). We study the convergence of this formulation and the behaviour of the numerical solution as the absorption parameter $\nu$ tends to zero. The experiments in this section were performed with our code written in Octave. We implemented the scheme described in Section 2.2 for a case of $P_{1}$-space used for the approximation of $E_{x}$ and $E_{y}$ and $\theta=0$, thus working with System (14). We apply permutation to the above system to obtain a 7-diagonal Hermitian matrix and solve the system with the Gauss back substitution algorithm. The intermediate part briefly validates the semi-lagrangian discretization of the time domain formulation. The last part of the section deals with the question of the equivalence of the limiting absorption and limited amplitude principle. We compare the solutions obtained as $\nu \rightarrow 0$ with the help of the frequency and time domain codes (computed for large values of time). The time-domain code implements the scheme described in Section 3.1 and is written in Fortran. 


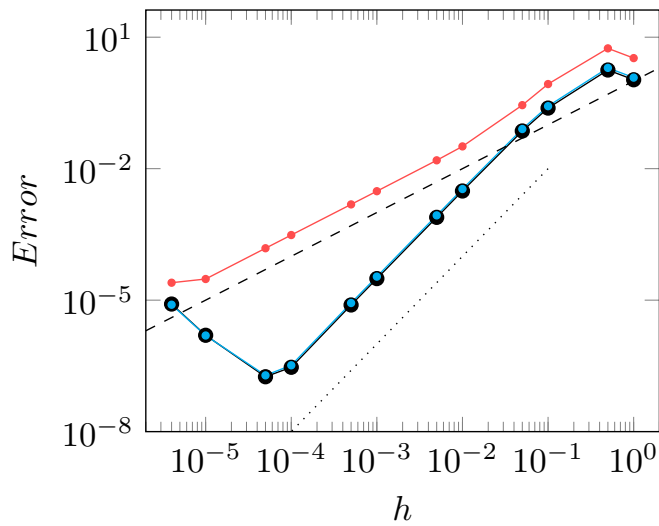

$\multimap\left\|E_{x}-E_{x}^{c}\right\|_{L^{2}}$
$\longrightarrow\left\|E_{y}-E_{y}^{c}\right\|_{H^{1}}$
$\because\left\|E_{y}-E_{y}^{c}\right\|_{L^{2}}$
$\cdots \cdots\left(h^{2}\right)$
$--O(h)$

FiguRE 3. Convergence rates for the problem described in Section 4.1.1 (the problem 10 ) discretized as (14) with the parameters $(15)$ and the boundary condition (16)). Here $E_{y}^{c}(x)=$ $\operatorname{Ai}(x)$ and $E_{x}^{c}(x)=i \frac{\delta(x)}{\alpha(x)} \operatorname{Ai}(x)$.

\subsection{Frequency Domain Problem}

\subsubsection{Validity of the Implementation}

To check the validity of the code, we first perform a numerical experiment in the following case:

$$
\alpha(x)=x^{2}+1, \quad \delta(x)=-\left(\alpha^{2}+x \alpha\right)^{\frac{1}{2}}, \quad \nu=0, \quad \theta=0 .
$$

In this case $E_{y}$ that solves 10 satisfies the Airy equation, see also Remark 2.4 .

$$
E_{y}^{\prime \prime}-x E_{y}=0, \quad x \in[-L, H]
$$

The physical solution of this equation, the Airy function $\operatorname{Ai}(x)$, see [1, Chapter 10.4], is analytic and decays rapidly as $x \rightarrow \infty$. Hence, choosing $H$ sufficiently large (the actual value used in the experiments $H=10$, where $\left.\left|\mathrm{Ai}^{\prime}(10)\right|<1 e-9\right)$ and the boundary conditions

$$
\partial_{x} E_{y}(-L)+2 i E_{y}(-L)=\operatorname{Ai}^{\prime}(-L)+2 i \operatorname{Ai}(-L), \quad \partial_{x} E_{y}(H)=0 \approx \operatorname{Ai}^{\prime}(H),
$$

the exact solutions are: $\operatorname{Ai}(x)$ for the unknown $E_{y}$, and $i \frac{\delta(x)}{\alpha(x)} \operatorname{Ai}(x)$ for $E_{x}$. Hence both functions are smooth. The well-posedness of the respective variational formulation is due to Remark 2.4 .

In Fig. 3 we verify the convergence rates for this problem, comparing the solution with the analytic solutions. Importantly, the obtained convergence rates are in agreement with known estimates of the standard theory of convergence [2, Chapter 5.7, Chapter 0.4], provided that the Aubin-Nitsche lemma [4, Theorems 3.2.4, 3.2.5] holds for the variational formulation.

\subsubsection{Solution of the Frequency-Domain Problem with Resonance}

Let us consider the case of the resonance, more precisely, we choose sufficiently smooth $\alpha, \delta$, so that $\alpha(0)=0$ and $\delta(0) \neq 0$, and the solvability conditions of Lemma 2.3 are satisfied. For simplicity, let us assume that $\alpha(x)$ is smooth and

$$
\alpha(x)=-x \text { in some neighbourhood of } 0 .
$$


Let $h>0$ denote the meshwidth. Given $\mathbf{V}_{h}=P_{h}^{1} \times P_{h}^{1}$, with $P_{h}^{1}$ consisting of piecewise-linear (hat) functions, and $E_{x}^{\nu}, E_{x}^{\nu, h}$ being respectively the exact and the numerical solution, we look for the ratio $h(\nu)$ that would ensure the bound on the absolute error

$$
\left\|E_{x}^{\nu}-E_{x}^{\nu, h}\right\|_{L^{2}}<\epsilon
$$

given a fixed value of $\epsilon>0$ and $\nu \rightarrow 0$. Without loss of generality here we assume that $\nu>0$.

We use the following ingredients:

- The Céa lemma applied to the problem $(12)$; here $\mathbf{E}^{\nu}$ is the exact solution, $\mathbf{E}^{\nu, h}$ is the computed solution, and $C_{c}$ and $C_{i}$ are respectively the continuity and the coercivity constants:

$$
\left\|\mathbf{E}^{\nu}-\mathbf{E}^{\nu, h}\right\|_{\mathbf{v}} \leq \frac{C_{c}}{C_{i}} \min _{\mathbf{v} \in \mathbf{V}_{h}}\left\|\mathbf{E}^{\nu}-\mathbf{v}\right\|_{\mathbf{v}} \leq C \nu^{-1} \min _{\mathbf{v} \in \mathbf{V}_{h}}\left\|\mathbf{E}^{\nu}-\mathbf{v}\right\|_{\mathbf{v}}, C>0 .
$$

The last inequality follows from Lemma 2.3 and is valid for $\nu \rightarrow 0$.

- The estimate from [2, Chapter 0] on the rate of convergence of the interpolation

$$
\begin{array}{r}
\left\|v-I^{h} v\right\|_{L^{2}} \leq C_{1} h^{2}\left\|v^{\prime \prime}\right\|_{L^{2}}, C_{1}>0 \\
\left\|v-I^{h} v\right\|_{H^{1}} \leq C_{2} h\left\|v^{\prime \prime}\right\|_{L^{2}}, C_{2}>0,
\end{array}
$$

where $I^{h}$ is an interpolation operator onto $P_{h}^{1}$.

- An explicit bound on $\left\|\left(E_{x}^{\nu}\right)^{\prime \prime}\right\|_{L^{2}(\Omega)}$ based on 10] and 9. Proposition 1]. First of all, we will assume that the main contribution to $\left\|\left(E_{x}^{\nu}\right)^{\prime \prime}\right\|_{L^{2}(\Omega)}$ comes from the behaviour in the vicinity of the resonance, where, for sufficiently small $\nu$, the following expansion is valid 9 , Proposition 1]

$$
\begin{array}{r}
E_{x}^{\nu}(x)=\tilde{E}_{x}^{\nu}+S_{1}^{\nu}(x), \\
\tilde{E}_{x}^{\nu}=f_{\nu}(x) \log \rho_{\nu}\left(x-X_{\nu}\right)+\frac{g_{\nu}(x)}{\rho_{\nu}\left(x-X_{\nu}\right)},
\end{array}
$$

where $f_{\nu}, g_{\nu}, S_{1}^{\nu}$ are smooth functions, which, together with their derivatives, are bounded independently of $\nu$ for all sufficiently small values of $\nu ; \rho_{\nu}$ is a smooth function s.t. $\rho_{\nu}(0)=0$, with the derivative $\rho_{\nu}^{\prime}(0)=1$, the point $X_{\nu} \approx i \nu c+O\left(\nu^{2}\right)$, for some $c \in \mathbb{R} \backslash\{0\}$.

- An explicit bound on $\left\|\left(E_{y}^{\nu}\right)^{\prime \prime}\right\|_{L^{2}(\Omega)}$ based on [9. As before, we will assume that the main contribution to $\left\|\left(E_{y}^{\nu}\right)^{\prime \prime}\right\|_{L^{2}(\Omega)}$ comes from the behaviour in the vicinity of the resonance, where, for sufficiently small $\nu$, it holds [9, Proposition 1]

$$
\begin{array}{r}
E_{y}^{\nu}(x)=\tilde{E}_{y}^{\nu}(x)+S_{2}^{\nu}(x), \\
\tilde{E}_{y}^{\nu}(x)=(\alpha(x)+i \nu) p_{\nu}(x) \log \rho_{\nu}\left(x-X_{\nu}\right),
\end{array}
$$

where $\rho_{\nu}, X_{\nu}$ are is in 21), and $p_{\nu}, S_{2}^{\nu}$ are smooth functions, which, together with their derivatives, are bounded independently of $\nu$ for all sufficiently small values of $\nu$.

Let us recall that we defined the function space $\mathbf{V}=L^{2}(\Omega) \times H^{1}(\Omega)$. Estimate 190 combined with 20 results in, for some $C>0$.

$$
\left\|E_{x}^{\nu}-E_{x}^{\nu, h}\right\|_{L^{2}} \leq C \nu^{-1} h\left\|\left(E_{y}^{\nu}\right)^{\prime \prime}\right\|_{L^{2}}+C \nu^{-1} h^{2}\left\|\left(E_{x}^{\nu}\right)^{\prime \prime}\right\|_{L^{2}} .
$$


First of all let us examine the behaviour the derivatives of $E_{y}^{\nu}$ in the vicinity of the resonance, in particular, the term $\tilde{E}_{y}^{\nu}$ :

$$
\left(\tilde{E}_{y}^{\nu}\right)^{\prime}=\alpha^{\prime}(x) p_{\nu}(x) \log \rho_{\nu}\left(x-X_{\nu}\right)+(\alpha(x)+i \nu) p_{\nu}^{\prime}(x) \log \rho_{\nu}\left(x-X_{\nu}\right)+\frac{(\alpha(x)+i \nu) p_{\nu}(x) \rho_{\nu}^{\prime}\left(x-X_{\nu}\right)}{\rho_{\nu}\left(x-X_{\nu}\right)} .
$$

The last term in this expression is well-defined in $x=0$, even for $\nu=0$ (the limit can be obtained with the help of the L'Hopital's rule and recalling that $\rho_{\nu}\left(x-X_{\nu}\right)=x-X_{\nu}+O\left(\left(x-X_{\nu}\right)^{2}\right)$ in the vicinity of the resonance), and is uniformly bounded for sufficiently small $\nu$, together with its derivatives. The second term is also well-defined in $x=0$. To examine the behaviour of $\left(\tilde{E}_{y}^{\nu}\right)^{\prime \prime}$, it is sufficient that we study solely the singular first term of 23

$$
\left(\alpha^{\prime}(x) p_{\nu}(x) \log \rho_{\nu}\left(x-X_{\nu}\right)\right)^{\prime}=\frac{\alpha^{\prime}(x) p_{\nu}(x) \rho_{\nu}^{\prime}\left(x-X_{\nu}\right)}{\rho_{\nu}\left(x-X_{\nu}\right)}+\left(\alpha^{\prime}(x) p_{\nu}(x)\right)^{\prime} \log \rho_{\nu}\left(x-X_{\nu}\right) .
$$

The term $\frac{\alpha^{\prime}(x) p_{\nu}(x) \rho_{\nu}^{\prime}\left(x-X_{\nu}\right)}{\rho_{\nu}\left(x-X_{\nu}\right)}$ can be bounded, for all sufficiently small $|x|$, by

$$
\left|\frac{\alpha^{\prime}(x) p_{\nu}(x) \rho_{\nu}^{\prime}\left(x-X_{\nu}\right)}{\rho_{\nu}\left(x-X_{\nu}\right)}\right| \leq \frac{C}{|x-i \nu c|}, C>0
$$

where we used an explicit form of $\rho_{\nu}\left(x-X_{\nu}\right)=x-X_{\nu}+O\left(\left(x-X_{\nu}\right)^{2}\right)$ valid for small $\nu$ and $|x|$, as well as the expansion $X_{\nu}=i c \nu+O\left(\nu^{2}\right)$. For sufficiently small $\nu$, the term $\left(\alpha^{\prime}(x) p_{\nu}(x)\right)^{\prime} \log \rho_{\nu}\left(x-X_{\nu}\right)$ can be bounded by the above expression as well.

Therefore, assuming that the main contribution to $\left\|\left(E_{y}^{\nu}\right)^{\prime \prime}\right\|_{L^{2}(\Omega)}$ comes from the vicinity of the resonance, where for $\nu=0\left(E_{y}^{\nu}\right)^{\prime \prime}$ is singular, we obtain the following rough bound

$$
\left\|\left(E_{y}^{\nu}\right)^{\prime \prime}\right\|_{L^{2}(\Omega)} \leq G+G^{\prime}\left(\int_{-L}^{H} \frac{1}{|x-i \nu c|^{2}} d x\right)^{\frac{1}{2}} \leq \frac{C}{\sqrt{\nu}}, \quad G, G^{\prime}, C>0,
$$

for sufficiently small $\nu>0$.

To derive a similar bound on the second derivative of $E_{x}^{\nu}$, we assume that the main contribution to $\left\|\left(E_{x}^{\nu}\right)^{\prime \prime}\right\|_{L^{2}(\Omega)}$ comes from the singular part of $\tilde{E}_{x}^{\nu}$, whose second derivative can be represented in the following form, for some functions $F_{\nu, k}(x), k=0, . ., 3$, uniformly bounded in $x, \nu$ in the vicinity of $(x, \nu)=(0,0)$ :

$$
\frac{d^{2} \tilde{E}_{x}^{\nu}}{d x^{2}}=\sum_{k=1}^{3} \frac{F_{\nu, k}(x)}{\left(\rho_{\nu}\left(x-X_{\nu}\right)\right)^{k}}+F_{\nu, 0}(x) \log \rho_{\nu}\left(x-X_{\nu}\right) .
$$

Then there exists $c>0$, s.t. for all sufficiently small $\nu$

$$
\left\|\frac{d^{2} E_{x}^{\nu}}{d x^{2}}\right\|_{L^{2}}^{2} \leq c \int_{-L}^{H} \frac{1}{\left(x^{2}+\nu^{2}\right)^{3}} d x \leq C \nu^{-5}
$$

where $C>0$ does not depend on $\nu$. Plugging this into 22 we obtain

$$
\left\|E_{x}^{\nu}-E_{x}^{\nu, h}\right\|_{L^{2}} \leq C \nu^{-\frac{7}{2}} h^{2}+C \nu^{-\frac{3}{2}} h .
$$


From this it thus follows that to ensure 18 should be chosen as

$$
h=\alpha_{\epsilon} \nu^{\frac{7}{4}}
$$

where $\alpha_{\epsilon}>0$ depends on $\epsilon$ but does not depend on $\nu$. This prediction is very severe and is due to the singular nature of the problem under consideration.

Let us check numerically whether this holds true. To do so, we conduct the numerical experiment with parameters for Problem (10) as given in Table 1. Recall as well that $\theta=0$.

\begin{tabular}{|c|c|}
\hline Parameter & Value \\
\hline$\alpha(x)$ & $\left\{\begin{array}{lr}10, & x \leq-10 \\
-x, & -10<x \leq 5 \\
-5, & x>5\end{array}\right.$ \\
\hline$\delta(x)$ & $\left\{\begin{array}{lr}0, & x \leq-10 \\
-4 / 30 x-4 / 3, & -10<x \leq 5 \\
-2, & x>5\end{array}\right.$ \\
\hline$g^{i n c}(-L)$ & $-2 \sqrt{2} i \exp (-22 \sqrt{2} i)$ \\
\hline$\lambda$ & $\sqrt{10}$ \\
\hline$L$ & 15 \\
\hline$H$ & 10 \\
\hline
\end{tabular}

TABLE 1. The parameters for the problem with the resonance.

Denoting by $E_{x}^{\nu, h}$ the solution $E_{x}(x)$ computed on the mesh with width $h$ and absorption $\nu$, and by $E_{x}^{\nu}$ the solution computed on a mesh multiple times finer (more precisely, the reference mesh was typically 10-200 times finer, except when the computation was performed for very small values of $h$ when results were compared with results computed on twice finer meshes), let

$$
h_{\epsilon}=\sup \left\{h:\left\|E_{x}^{\nu, h^{\prime}}-E_{x}^{\nu}\right\|<\epsilon \text { for all } h^{\prime}<h\right\} .
$$

The computed dependence of $h_{\epsilon}$ on $\nu$ is shown in Fig. 4 . We can see that Estimate 25 is pessimistic compared to the one suggested by (26), at least for given values of $\epsilon$ and for a chosen range of $\nu>0$.

Remark 4.1. It can be shown that $\left\|E_{x}^{\nu}\right\|_{L^{2}} \leq \frac{C}{\sqrt{\nu}}, C>0$, and thus the relative error control

$$
\frac{\left\|E_{x}^{\nu}-E_{x}^{\nu, h}\right\|_{L^{2}}}{\left\|E_{x}^{\nu}\right\|_{L^{2}}} \leq \epsilon
$$

is ensured by choosing $h$ as $\beta_{\epsilon} \nu^{\frac{3}{2}}, \beta_{\epsilon}>0$.

In Figure 5 we demonstrate the dependence of the condition number of the matrix of System (14) on $\nu$, for several values of $h$. Remarkably, for $\nu=0$ the computed matrices are not singular. We do not know the exact reason for this. As an additional illustration, we compare the solutions for very small $\nu$ and $\nu=0$ for different meshes in Fig. 5 .

\subsection{A validation of the semi-lagrangian scheme}

With very basic simulation, we show that the numerical strategy based on co-localised semi-lagrangian discretization is valid even for the resonance. The numerical tests have been kept to a minimum, and many more are needed to fully validate the concept. The setting of the numerical results of Figure 6 is the following. We use a 7 th order semi-lagrangian scheme, and the CFL is $\nu=0.5$. The peak is the hybrid resonance, visible 

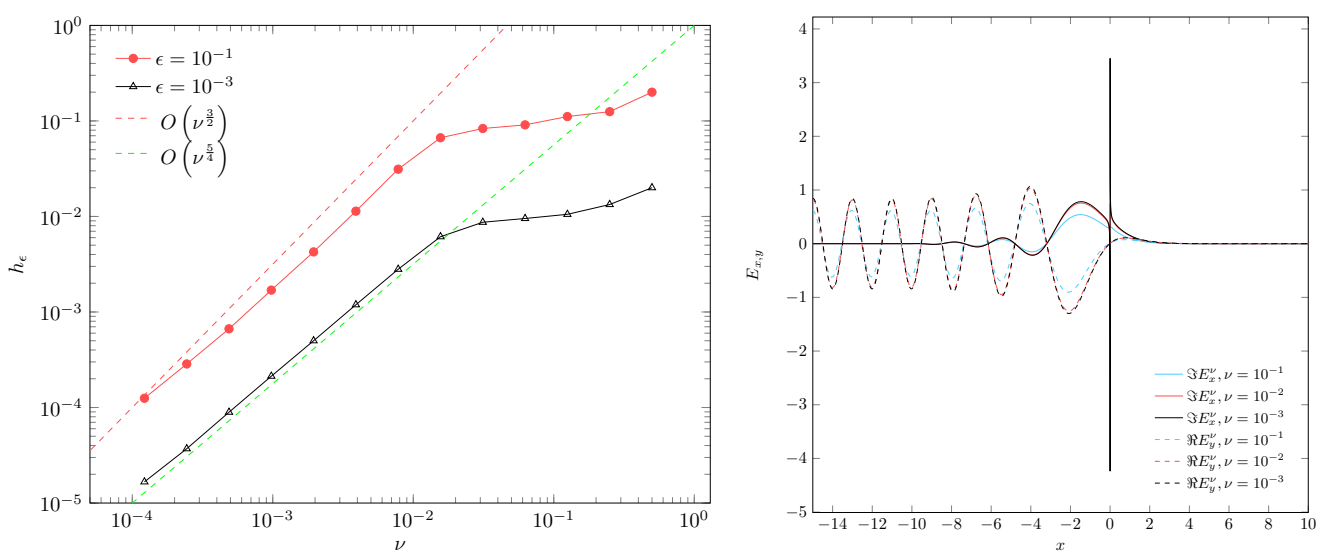

Figure 4. We consider the problem 10 with the parameters defined in Table 1 . In the left figure the dependence of $h_{\epsilon}$ as defined by (26) on $\nu$ is demonstrated. In the right figure we show the computed solutions for this problem (for brevity denoted by $E_{x, y}^{\nu}$ ) for different values of $\nu$.
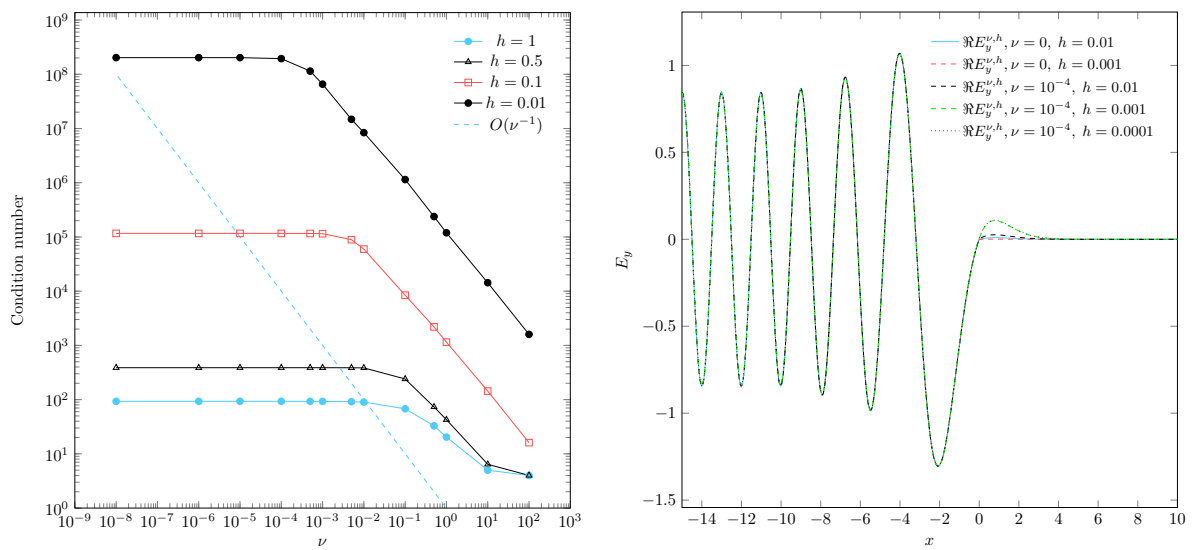

Figure 5. We consider the problem (10) with the parameters defined in Table 1 . The left plot demonstrates the dependence of the condition number of the system 114 on $\nu$, for different values of $h$ (the condition number for $\nu=0$ is not shown, however, for all values of $h$ as shown in the plot, the matrix was non-singular even for $\nu=0$ ). The right plot shows $E_{y}^{\nu, h}$ computed on different meshes for small $\nu$. It can be seen that for $h=10^{-2}$ and $h=10^{-3}$ the solutions computed for $\nu=0$ are almost indistinguishable.

on $E_{x}$ and $u_{x}$. The total energy $\mathcal{E}(t)$ is the physical energy. The comparison with Figures 4 and 12 shows that the singular nature of the resonance is captured by this new scheme without any doubt.

Notice that in this case we shifted the computational domain for visualization purposes, so that $L=0$ and the point where the resonance occurs is $x_{h}=20$. 

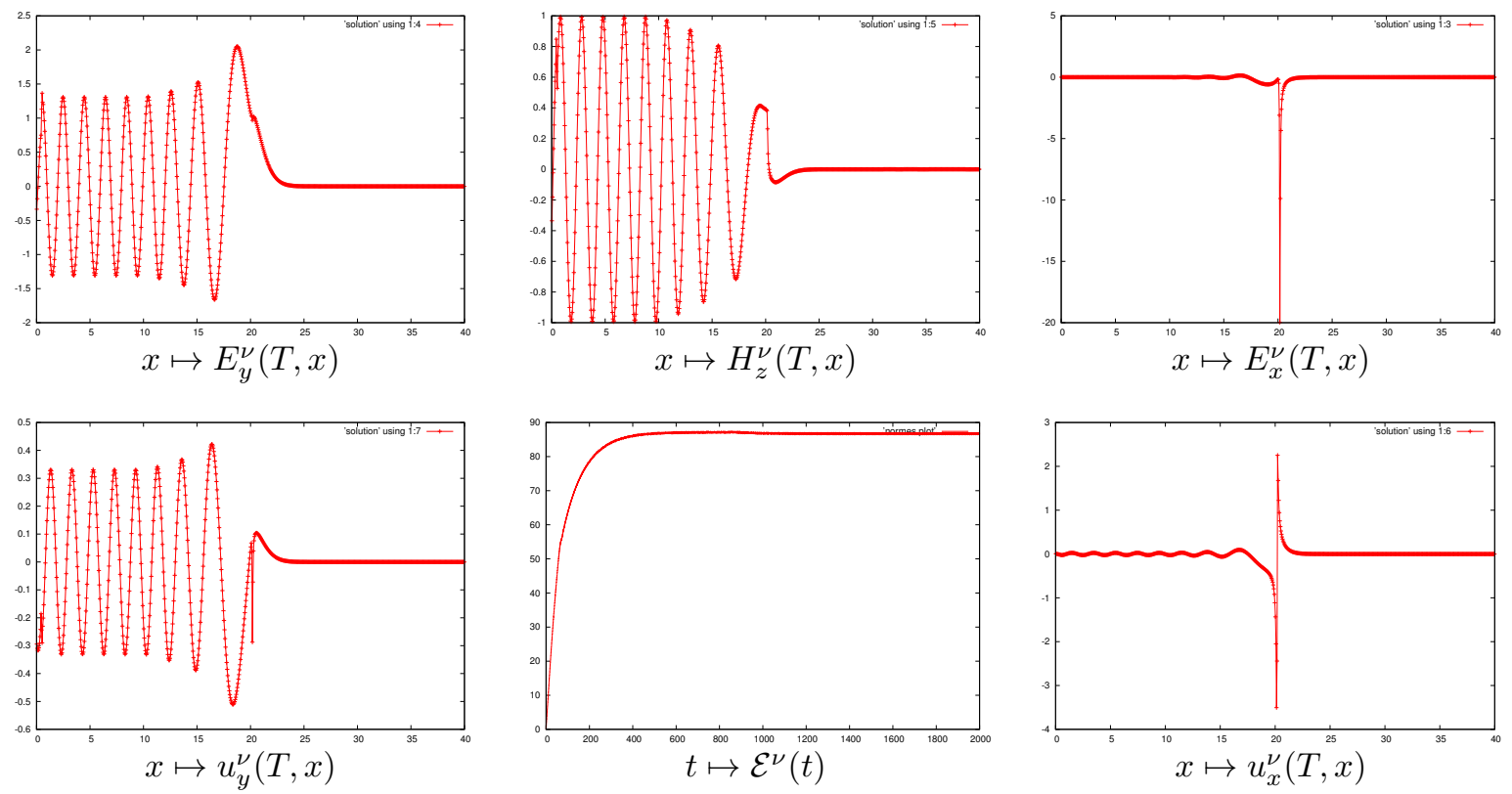

Figure 6. An antenna sends a time-harmonic wave on the left. The medium is propagative on the left and non-propagative on the right. The resonance is visible on the real functions $E_{x}^{\nu}$ and $u_{x}^{\nu}$. The time of the simulation $T=2000$ is chosen large enough so that the periodic solution is captured, at least numerically. The number of cells is typically 10000 to reach space convergence. The shift for visualization purposes if +20 , which explains why the resonance is now at $x=20$.

\subsection{Limiting Amplitude and Limiting Absorption Principle}

In this section we perform several numerical experiments to check whether the limit amplitude principle and the limit absorption principle are equivalent for our problem. That is we compare the solution of the time harmonic code with the solution of the time domain staggered code.

We normalize $\epsilon_{0}=\mu_{0}=1$ and $\omega=c=1$. Also, we set $m_{e}=1$ and $e=-1$. From this it follows that $w_{c}=-B_{0}$ and $w_{p}^{2}=N_{e}$. We consider the following two cases:

- case $N_{e} \neq$ const, no resonance;

- case $N_{e} \neq$ const, resonance.

For every fixed absorption rate $\nu>0$, in the time domain we choose the boundary conditions

$$
\begin{aligned}
\left.\partial_{t} H\right|_{x=-L} & =-\left.\partial_{x} E_{y}\right|_{x=-L}=G \sin (t), G \in \mathbb{R}, \\
\left.\partial_{t} H\right|_{x=H} & =0,
\end{aligned}
$$

and zero initial conditions, and in the frequency domain

$$
\left.\partial_{x} \hat{E}_{y}\right|_{x=-L}=G,\left.\partial_{x} \hat{E}_{y}\right|_{x=H}=0 .
$$


We compute the solution $\mathbf{E}^{\nu}(t)$ for large $t$ in the time domain, and the solution $\hat{\mathbf{E}}^{\nu}$ in the frequency domain. In the numerical experiments we check whether the following two quantities

$$
\lim _{t \rightarrow+\infty} \mathbf{E}^{\nu}(t), \text { and } \Im\left(\hat{\mathbf{E}}^{\nu} \mathrm{e}^{-i t}\right)
$$

are close as $\nu \rightarrow 0$ (provided that the first of these quantities exists).

In all the experiments in this section the CFL number was set to 0.5.

\subsubsection{No-Resonance Case}

We choose the parameters so that in the frequency domain, for the limiting amplitude problem, $\hat{E}_{y}^{\nu}, \nu=0$, satisfies the Airy equation, c.f. also Remark 2.4. We set $\omega_{c}=0($ thus $\delta(x)=0), \omega=1\left(\right.$ hence $\alpha(x)=1-N_{e}(x)$ ), on the domain as $(-0.5,10)$ and set the electron density $N_{e}(x)=1+x>0, x \in \Omega$. Importantly, since $\delta(x)=0$, in this case no resonance occurs and $E_{x}(x, t) \equiv 0$. This corresponds to the case when the tensor of dielectric permittivity $\underline{\underline{\varepsilon_{\omega}^{0}}}$ is diagonal.

The boundary conditions in $(27)$ are chosen as $G=A i^{\prime}(-0.5)$.

First we set $\nu=10^{-2}$. To demonstrate that the limiting amplitude principle indeed holds, we fix a point $x=x_{c}$ inside the domain and plot the dependence of the solution $E_{y}^{\nu}\left(x_{c}, t\right)$ on time $t$ for a range of $t \gg 1$ in Fig. 7. We compare this solution to the computed $\Im\left(\hat{E}_{y}^{\nu} \mathrm{e}^{-i t}\right)$, for fixed values of $t$. Both solutions appear to be in close agreement.

Fig. 8 (left) shows the solutions at a fixed point in space for $\nu=10^{-4}$. As previously, we fix a point $x=x_{c}$ inside the domain and plot the dependence of the solution $E_{y}^{\nu}\left(x_{c}, t\right)$ on time $t$ for a range of $t \gg 1$. One of our observations was that for smaller $\nu$ more time steps are required to achieve the limiting amplitude solution. For $\nu=10^{-4}$ we were not able to obtain the limiting amplitude solution for $t<3 \cdot 10^{4}$, unlike in the case of $\nu=10^{-2}$. For example, for $\nu=10^{-6}$ we were not able to reach the limiting amplitude solution even on the time interval $t \leq 1.92 e 6$, see Fig. 8 (right).
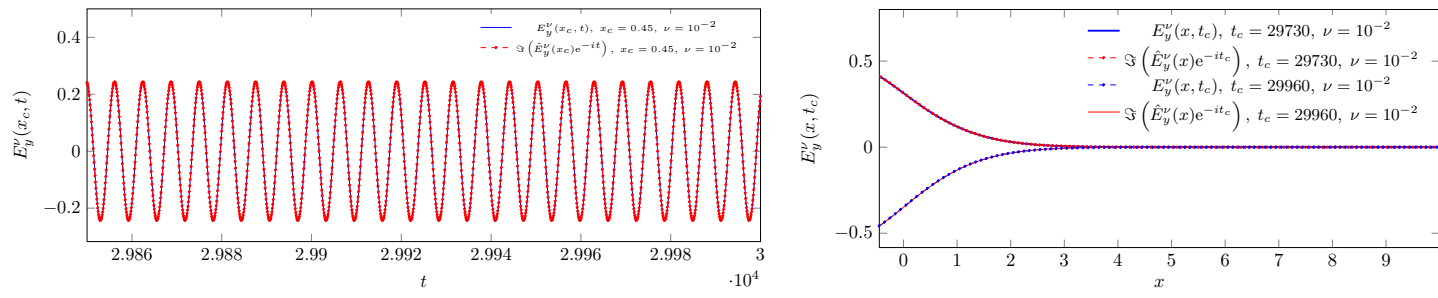

Figure 7. Dependence of the solution $E_{y}^{\nu}\left(x_{c}, t\right)$ to the problem described in Section 4.3.1 on time for large times is demonstrated in the left figure. In the right figure we show the solution to the same problem for $\nu=10^{-2}$, for two fixed moment of times.

\subsubsection{Resonance Case}

For the resonance case, we choose the parameters as in Table 2 . Since $\alpha(x)=\left(1-2 N_{e}(x)\right), \alpha(0)=0$, and clearly, $\delta(0) \neq 0$. We compare the results for $\nu=10^{-2}, 10^{-3}, 10^{-4}$ in Fig. 9, 10, 11, 12, 13. The harmonic dependence of the solution on time is demonstrated by fixing $x_{c}$ inside the domain and plotting $E_{x}^{\nu}\left(x_{c}, t\right)$ and $E_{y}^{\nu}\left(x_{c}, t\right)$ for a range of $t$.

Figures 11, 12, 13 show that in the case of the resonance the limiting amplitude solution is achieved, and both limiting amplitude and limited absorption solutions are in close agreement (but in the points close to a point where the resonance occurs). These numerical results show that the staggered scheme resolves the resonance, similarly to the semi-lagrangian scheme described before. Like in the regular case, longer computations are needed to achieve the limiting amplitude solution for smaller $\nu$. 

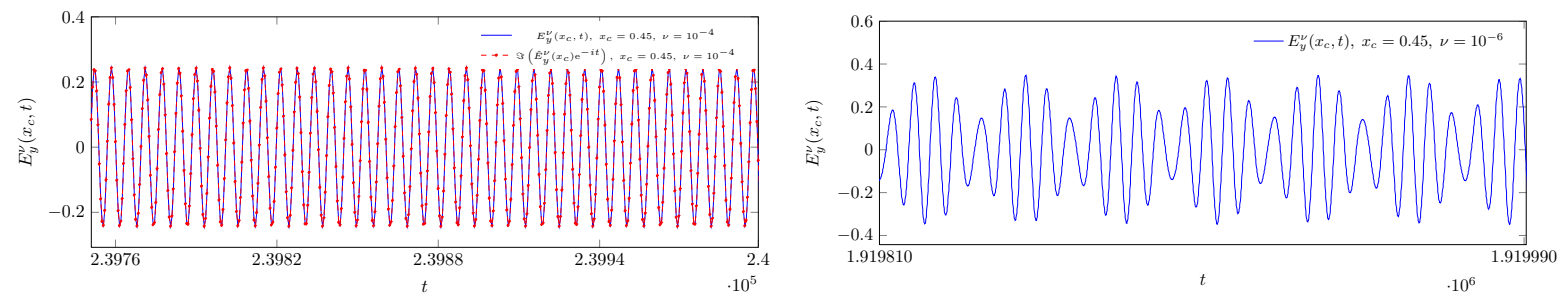

Figure 8. In the left figure we plot the dependence of the solution $E_{y}^{\nu}\left(x_{c}, t\right)$ to the problem described in Section 4.3.1 on time $t$, with $\nu=10^{-4}$ and $x_{c}=0.45$. In the right figure we show the solution to the same problem for $\nu=10^{-6}$ at the same point $x_{c}$, for larger times. As we can see, for $\nu=10^{-4}$ the limiting amplitude solution was reached for large $t$. For $\nu=10^{-6}$ we were not able to obtain the limiting amplitude solution even for $t \approx 1.9 \mathrm{e} 6$.

\begin{tabular}{|c|c|c|}
\hline Parameter & Value & \\
\hline$L$ & 5 & \\
\hline$H$ & 19 & \\
\hline$\omega_{c}$ & $\sqrt{0.5}$ & \\
\hline$N_{e}(x)$ & $\left\{\begin{array}{l}0.25, \\
\frac{1+x}{2},\end{array}\right.$ & $\begin{array}{r}x<-0.5, \\
x \geq-0.5, x \leq 9\end{array}$ \\
\hline$G$ as in 27$)$ & $\begin{array}{c}5,5, \\
0.11\end{array}$ & $x>9$ \\
\hline
\end{tabular}

TABle 2. Parameters for numerical simulations in Section 4.3.2
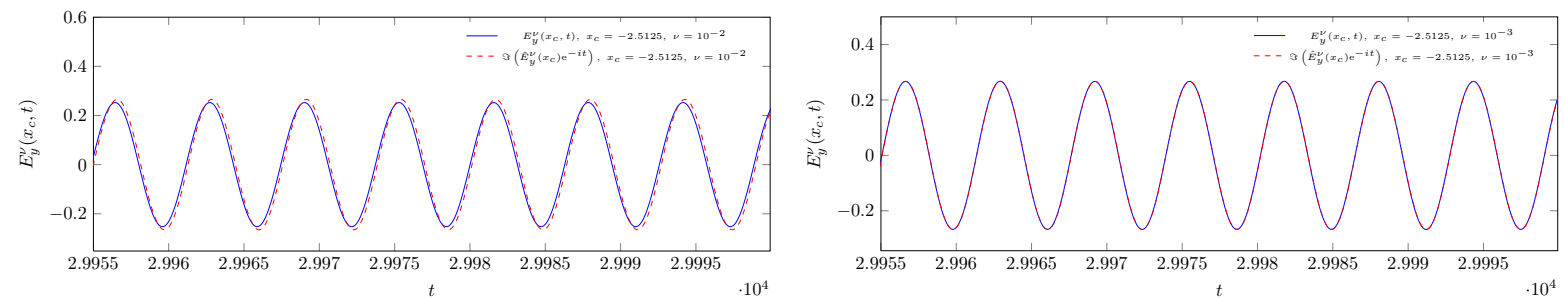

Figure 9. The figures demonstrate the dependence of the solutions to the problem described in Section $4.3 .2 E_{y}^{\nu}(-2.5125, t)$ on time $t$ for different values of $\nu$.

\section{Conclusions}

In this work we considered the frequency and the time-domain formulations of the $X$-mode Maxwell equations. In particular, we proved the well-posedness of the regularized variational formulation with the absorption parameter $\nu$, as well as studied the convergence of the finite element method for the problem with the resonance. The piecewise-linear FEM approximates the resonant solution rather well, at least for moderate values of $\nu$, however, the discretization size should be chosen roughly proportional to $\nu^{\frac{7}{4}}$, in order to obtain an accurate discretization. Indeed, it would be interesting to look at the convergence of the adaptive finite elements for this kind of problems. Another unanswered question is the well-posedness of the discrete problem when the continuous problem is ill-posed. We have demonstrated by numerical means that while the condition number of the FEM matrix grows, the matrix remains invertible even for $\nu=0$.

We proposed two different schemes for solving the time-dependent problem. Our numerical experiments demonstrate that both of them capture the singular behaviour of the solutions in the resonant case. 


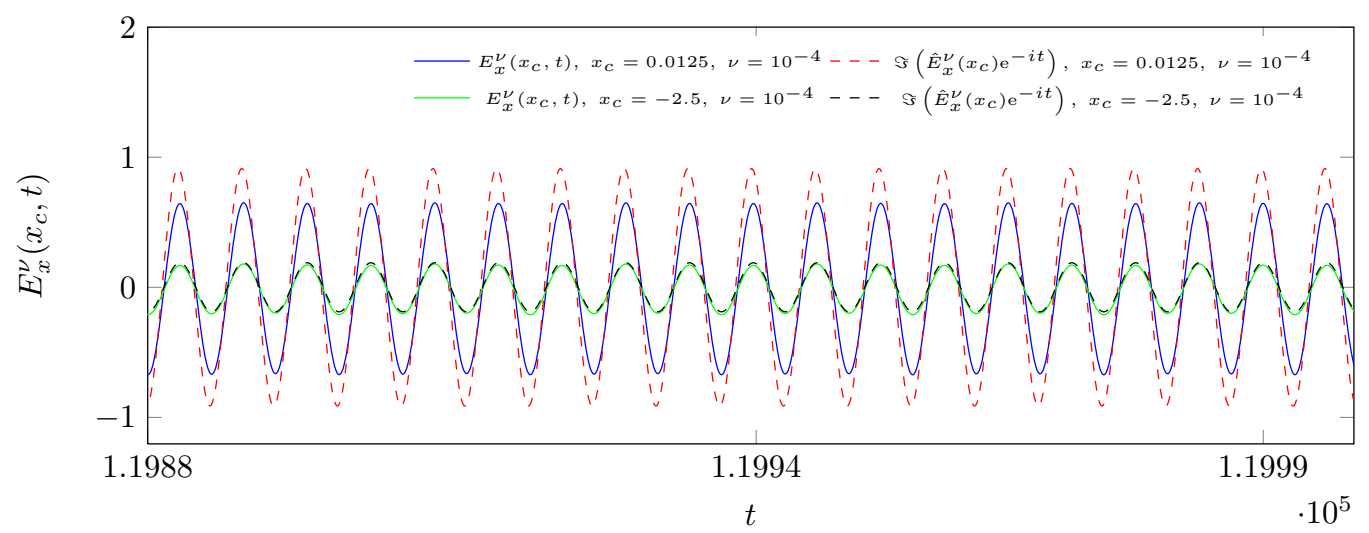

Figure 10. The plot shows the dependence of the solution $E_{x}^{\nu}\left(x_{c}, t\right), \nu=10^{-4}$, to the problem described in Section 4.3.2 on time $t$ for fixed $x_{c}=0.0125$ and for $x_{c}=-2.5$. We can see that the solutions computed in the time domain are in close agreement with the solution computed in the frequency domain at the point $x_{c}=-2.5$, however, differ at the point 0.0125 which is close to $x=0$ where the resonance occurs.
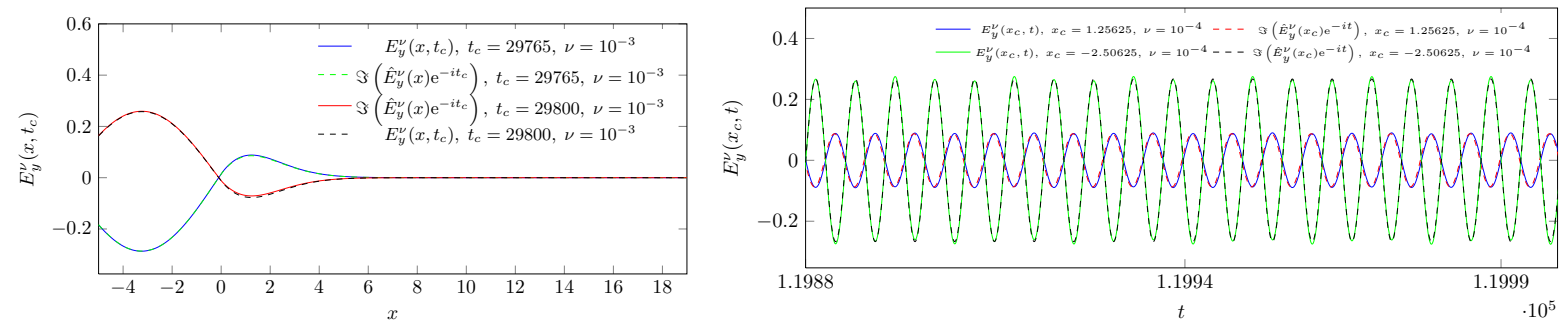

Figure 11. The left figure demonstrates the dependence of the solution $E_{y}^{\nu}(x, t)$ to the problem described in Section 4.3.2 on $x$ for fixed values of $t$. For a chosen value of $\nu$ the solutions computed in the time domain are in close agreement to the solutions computed in the frequency domain. This seems to be only partially true for the computed values $E_{x}^{\nu}(x, t)$ in this case (which may be caused by the discretization). The right figure shows the dependence of the solution $E_{y}^{\nu}(x, t)$ to the same problem for $\nu=10^{-4}$ on time for fixed $x=x_{c}$. We can see that the limiting amplitude solution had been achieved, and the difference between the solutions computed in the time and frequency domain is not visible on this scale (c.f. Figure 13).

The other part of the experiments concerned the equivalence of the limiting absorption and limiting amplitude solutions. We have shown that for small $\nu$ and large times the solution computed in the time domain is close to the solution predicted by the limiting amplitude principle; as $\nu \rightarrow 0$, the solution oscillates harmonically as $t \rightarrow+\infty$, however, we were not able to compute the limiting amplitude solution for very small values of $\nu$.

\section{REFERENCES}

[1] Abramowitz, M. and Stegun, I. A., eds. (1972). Handbook of Mathematical Functions with Formulas, Graphs, and Mathematical Tables. New York: Dover Publications. Handbook of mathematical functions

[2] Brenner, S. C. and Scott, R. (2008). The mathematical theory of finite element methods (Vol. 15). Springer.

[3] Charles, F., Després, B., Mehrenberg, M. (2013). Enhanced convergence estimates for semi-lagrangian schemes Application to the Vlasov-Poisson equation, SIAM J. Numer. Anal., 51(2), 840-863.

[4] Ciarlet, P. G. (1978). The Finite Element Method For Elliptic Problems. North-Holland Publishing Company. 

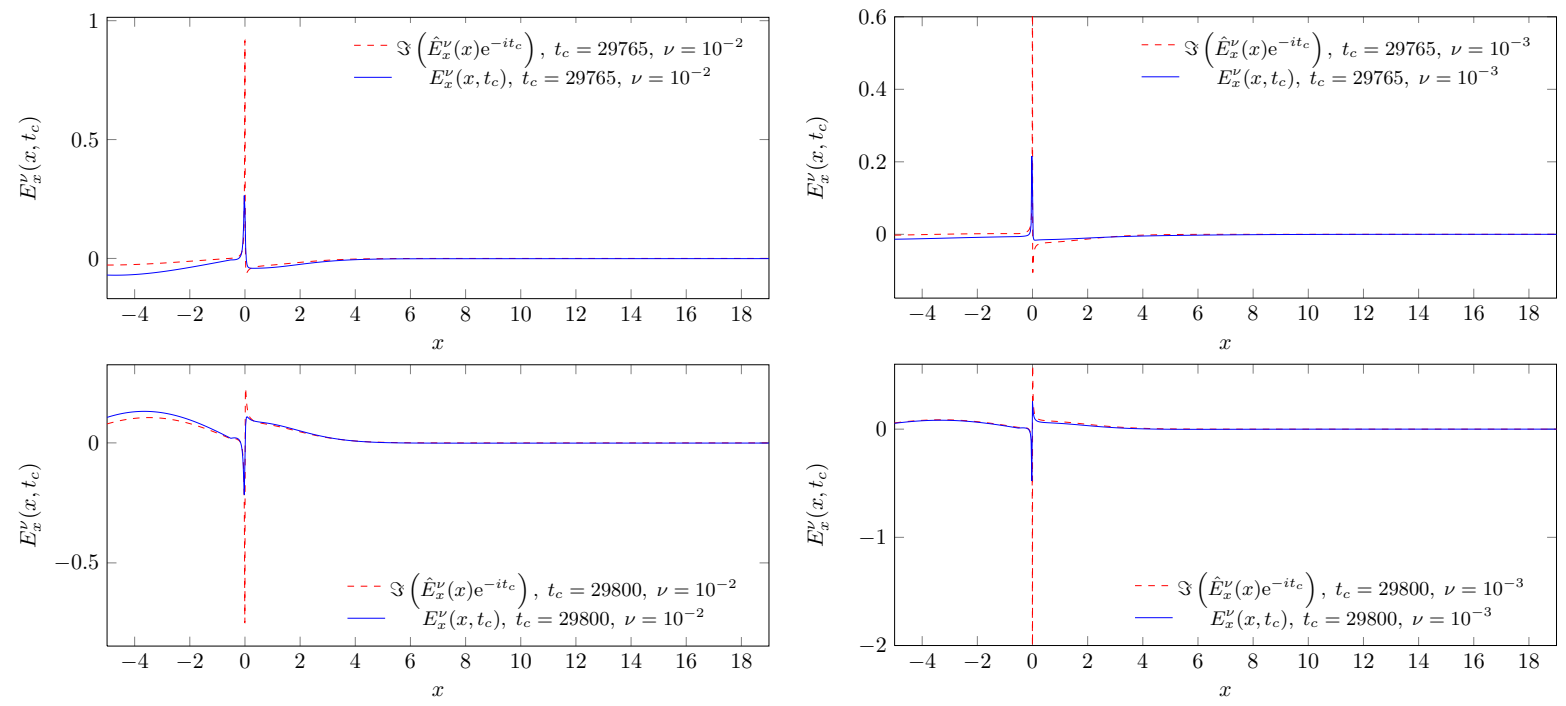

Figure 12. The figures demonstrate the dependence of the solutions $E_{x}^{\nu}(x, t)$ to the problem described in Section 4.3 .2 on $x$ for fixed values of $t$.
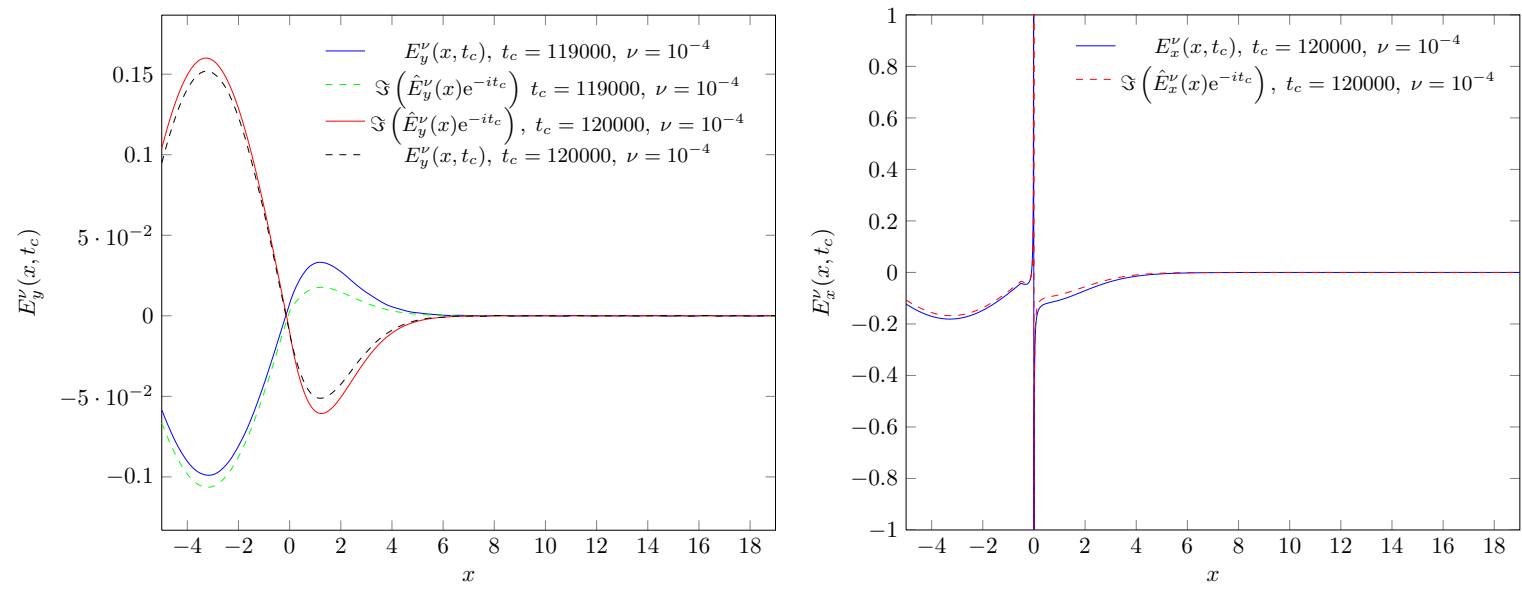

Figure 13. The figures demonstrate the dependence of the solutions $E_{x}^{\nu}(x, t)$ and $E_{y}^{\nu}(x, t)$ to the problem described in Section 4.3.2 on $x$ for fixed values of $t$. While the solution in the time-domain in the resonance point $x=0$ has a smaller absolute value than that computed in the frequency domain (in the plot both solutions are truncated to axis limits), in other points the values are rather close.

[5] Da Silva, F., Campos-Pinto, M., Després, B., and Heuraux, S. (2014). Stable coupling of the Yee scheme with a linear current model.

[6] Da Silva, F., Heuraux, S., Gusakov, E. Z., and Popov, A. (2010). A Numerical Study of Forward-and Backscattering Signatures on Doppler-Reflectometry Signals. Plasma Science, IEEE Transactions on, 38(9), 2144-2149.

[7] Da Silva, F., Heuraux, S. and Manso, M. (2006). Developments on reflectometry simulations for fusion plasmas: application to ITER position reflectometers. Journal of plasma physics, 72(06), 1205-1208.

[8] Del Pino, S., Després, B., Havé, P., Jourdren, H., and Piserchia, P. F. (2009). 3D finite volume simulation of acoustic waves in the earth atmosphere. Comput. \& Fluids, 38(4):765-777.

[9] Després, B., Imbert-Gérard, L.-M. and Lafitte, O. (2014). Singular solutions for the plasma at the resonance. 〈hal-01097364〉 
[10] Després, B., Imbert-Gérard, L. M. and Weder, R. (2014). Hybrid resonance of Maxwell's equations in slab geometry. Journal de Mathématiques Pures et Appliquées, 101(5), 623-659.

[11] Dumont, R. J., Phillips, C. K., and Smithe, D. N. (2005). Effects of non-Maxwellian species on ion cyclotron waves propagation and absorption in magnetically confined plasmas. Physics of Plasmas (1994-present), 12(4), 042508.

[12] Eidus, D.M. (1969). The principle of limit amplitude. Russian Mathematical Surveys, vol. 24, issue 3, 97-167.

[13] Feix, M. R., Bertrand, P., and Ghizzo, A. (1994). Eulerian codes for the Vlasov equation. Series on Advances in Mathematics for Applied Sciences 22, Kinetic Theory and Computing, pp. 45.

[14] Filbet, F., Sonnendrucker, E. (2003). Comparison of Eulerian Vlasov solvers, Computer Physics Communications, 150, pp. $247-266$

[15] Freidberg, J. P. (2007). Plasma physics and fusion energy. Cambridge University Press.

[16] Guo, W. and Qiu, J. - M. (2013). Hybrid semi-Lagrangian finite element-finite difference methods for the Vlasov equation, Journal of Computational Physics, 234, pp. 108-132.

[17] Hattori, T. (2014). Décomposition de domaine pour la simulation Full-Wave dans un plasma froid. Ph.D. Thesis, Université de Lorraine.

[18] Imbert-Gérard, L. M. (2013). Analyse mathématique et numérique de problèmes d'ondes apparaissant dans les plasmas magnétiques (Doctoral dissertation, Université Pierre et Marie Curie-Paris VI).

[19] Labrunie, S., Bertrand, P., Roche, J.-R., Back, A., and Hattori, T. Electromagnetic wave propagation and absorption in magnetised plasmas: variational formulations and domain decomposition, Hal preprint 2014, https://hal.archives-ouvertes.fr/hal01075137.

[20] Morawetz, C. S. (1962). The limiting amplitude principle. Communications on Pure and Applied Mathematics, 15(3), 349-361.

[21] Sonnendrucker, E., Roche, J., Bertrand, P., and A. Ghizzo (1998). The Semi-Lagrangian Method for the Numerical Resolution of Vlasov Equations. J. Comput. Phys., 149, pp. 201-220.

[22] Stix, T. H. (1992). Waves in plasmas. Springer.

[23] Umeda, T., Nariyuki, Y. and Kariya, D. (2012). A non-oscillatory and conservative semi-Lagrangian scheme with fourth-degree polynomial interpolation for solving the Vlasov equation, Computer Physics Communications,183, pp. 1094-1100.

[24] Xu, L., and Yuan, N. (2006). FDTD formulations for scattering from 3-D anisotropic magnetized plasma objects. Antennas and Wireless Propagation Letters, IEEE, 5(1), 335-338.

This is an Open Access article distributed under the terms of the Creative Commons Attribution License (http://creativecommons.org/licenses/by/4.0), which permits unrestricted use, distribution, and reproduction in any medium, provided the original work is properly cited. 